\title{
Surface acoustic waves in one-dimensional piezoelectric phononic crystals with symmetric unit cell
}

\author{
A. N. Darinskii ${ }^{1}$ and A. L. Shuvalov ${ }^{2}$ \\ ${ }^{1}$ Institute of Crystallography FSRC “Crystallography and Photonics,” Russian Academy of Sciences, Leninskii pr. 59, Moscow 119333, Russia \\ ${ }^{2}$ Université Bordeaux, CNRS, UMR 5295, 33405 Talence, France
}

(Received 17 June 2019; revised manuscript received 14 October 2019; published 8 November 2019)

\begin{abstract}
The paper studies the existence of surface acoustic waves in half-infinite one-dimensional piezoelectric phononic crystals consisting of perfectly bonded layers, which are arranged so that the unit cell is symmetric, i.e., is invariant with respect to inversion about its midplane. An example is a bilayered structure with exterior layer being half thinner than the interior layers of the same material. The layers may be generally anisotropic. The maximum possible number of surface acoustoelectric waves referred to a fixed wave number and a given full stop band is established for different types of electric boundary conditions at the mechanically free or clamped surface. In particular, it is proved that the phononic crystal-vacuum interface can support two surface waves in any full stop band. The same statement holds true in the case of a metallized surface of the crystal. This number is greater than that in a purely elastic case. In the presence of crystallographic symmetry, which decouples the sagittally and horizontally polarized surface waves, their separate admissible numbers are obtained. It is shown that the propagation along the normal to the surface is a special case, where the maximum number of surface waves is less than that along oblique directions.
\end{abstract}

DOI: 10.1103/PhysRevB.100.184303

\section{INTRODUCTION}

Throughout recent years, there has been a steady interest to the propagation of bulk and surface acoustic waves in phononic crystals [1-3]. In particular, much attention has been paid to the wave propagation in one-dimensional (1D) phononic crystals, otherwise termed superlattices, which represent periodic sequences of multilayers [4]. Reflection of bulk waves was investigated in piezoelectric [5-7] and solid-fluid superlattices [8-10], as well as in solid-solid and solid-fluid Fibonacci structures [11-14]. Much effort has been devoted to studying the surface acoustic waves (SAWs) in 1D elastic and piezoelectric phononic crystals. The largest amount of theoretical data was obtained for shear horizontally polarized SAWs [15-23]. Sagittally polarized two-partial SAWs [24-29] and fully coupled three-partial SAWs [30-32] were also investigated. Note parallel developments in modeling of SAWs in two-dimensional phononic crystals [33-45].

In practice, the SAWs in phononic crystals have to be sought numerically; however, the efficiency of this search can be enhanced due to the theoretical predictions. As is well known, the SAWs in a periodic structure are generally restricted to full stop bands (the spectral zones with no propagating modes). The question is whether and how many SAWs can exist in a stop band. The problem of SAW existence in halfinfinite 1D phononic crystals of general anisotropy composed of purely elastic and piezoelectric layers was considered in Refs. [46,47], respectively. It is clear that explicit solutions for the wave characteristics in terms of the material parameters are certainly out of reach; however, developing the methods of anisotropic piezoacoustics of homogeneous media established in Refs. $[48,49]$ has made possible to predict a maximum possible number of SAWs per full stop band. This number embraces the SAW occurrence in a given phononic crystal with arbitrary asymmetric arrangement of layers within a unit cell and in its "reversed" counterpart with an inverse ordering of layers in the unit cell. On the methodological side, we note that the theory of $[48,49]$ exploits the properties of the eigenvectors of the so-called Stroh matrix, whereas Refs. [46,47] use the properties of the eigenvectors of the transfer matrix derived from the Stroh matrices of the constituent layers.

The present paper is concerned with the case where the 1D phononic crystal has a symmetric unit cell such that is invariant with respect to inversion about its midplane. The required symmetry can be realized by a corresponding arrangement of odd number of one distinct and others pairwise identical layers in a period. For instance, this may be a half-infinite bilayered phononic crystal in which the thickness of the exterior layer is half of thickness of the interior layers of the same material. It is significant that the unit-cell symmetry modifies the algebraic properties of the transfer matrix and thereby essentially affects the analysis of the SAW existence problem. Thus this case has to be considered independently, i.e., the sought predictions on SAW existence cannot be extracted as a corollary the derivations of Ref. [47] developed for the case of an asymmetric unit cell. Note that a number of SAWs per a stop band established in the asymmetric case embraces their occurrence in the given structure and in its "reversed" modification [47]. Both arrangements are identical to each other in the symmetric case, hence the maximum possible number of SAWs must cannot be greater than a half of the above aggregate number in the asymmetric case. Apparently, this observation suggests that passing from nonsymmetric to symmetric setting may lead to SAW degeneracies inside the stop bands and maybe to their disappearance due to merging with the band edges. However, the number of SAWs, one way or another vanishing at such transition, may generally appear different, and so there is no a priori evidence that a simple 
guess of the permissible number of SAWs in the symmetric case, which is inferred directly from its evaluation [47] for the asymmetric case, cannot overestimate the actual result. Resolving this uncertainty on the grounds of rigorous analysis is the main motivation for the present paper. In addition, we also analyze the case of wave normal propagation across the phononic crystal, which is a special situation not considered so far.

The paper is organized as follows. Section II briefly introduces the transfer matrix of a 1D half-infinite phononic crystal. The existence of SAWs in stop bands under different boundary conditions is analyzed in Sec. III. The influence of crystallographic symmetry on the SAW occurrence and the case of the normal propagation are also treated here. A numerical example is considered in Sec. IV. The conclusions are presented in Sec. V. Appendix A recaps the governing system of equations of piezoacoustics. Appendices B and $\mathrm{C}$ discuss the necessary properties of the transfer matrix, the impedance and admittance of the half-infinite phononic crystal as well as the properties of the other matrices involved in considerations.

\section{TRANSFER MATRIX AND ITS EIGENVECTORS}

Consider a harmonic SAW propagating with frequency $\omega$ and wave number $k$ along the surface of a half-infinite 1D piezoelectric phononic crystal $y \geqslant 0$ built up of perfectly bonded piezoelectric layers with interfaces parallel to the surface $y=0$ (see an example in Fig. 1). Let $\mathbf{m}$ be the unit vector lying in parallel to the surface and $\mathbf{n}$ be the unit vector orthogonal to the surface. The mechanical displacement $\mathbf{u}$ coupled under the quasielectrostatic approximation with the electric potential $\varphi$ and the associated normal components of the mechanical stress $\hat{\sigma}$ and the electric displacement $\mathbf{D}$ may be sought as

$$
\begin{aligned}
\left(\begin{array}{l}
\mathbf{u}(\mathbf{r}, t) \\
\varphi(\mathbf{r}, t)
\end{array}\right) & =\left(\begin{array}{l}
\mathbf{a}(y) \\
\phi(y)
\end{array}\right) \mathrm{e}^{i(k x-\omega t)}, \\
\left(\begin{array}{c}
\hat{\boldsymbol{\sigma}}(\mathbf{r}, t) \mathbf{n} \\
\mathbf{D}(\mathbf{r}, t) \cdot \mathbf{n}
\end{array}\right) & =-i\left(\begin{array}{l}
\mathbf{l}(y) \\
d(y)
\end{array}\right) \mathrm{e}^{i(k x-\omega t)},
\end{aligned}
$$

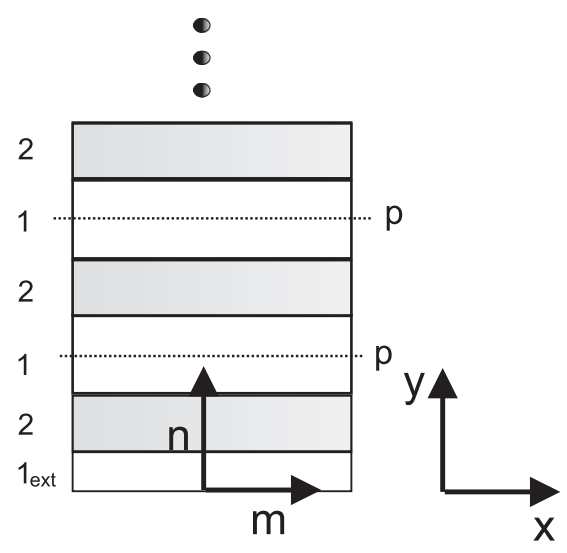

FIG. 1. Half-infinite phononic crystal with symmetric unit cell constructed of materials 1 (layers $1_{\text {ext }}$ and 1) and 2 (layers 2). The thickness of exterior layer $1_{\mathrm{ext}}$ is half the thickness of interior layers 1. Dotted lines $\mathrm{p}$ at the midline of layers 1 indicate the unit-cell edges. where $x=\mathbf{m} \cdot \mathbf{r}$ and $y=\mathbf{n} \cdot \mathbf{r}$. The vector of $y$-dependent amplitudes of the wave field (1), (2), arranged as either $\boldsymbol{\xi}_{\Phi}(y)=(\mathbf{a}, \phi, \mathbf{l}, d)^{t}$ or $\boldsymbol{\xi}_{F}(y)=(\mathbf{a}, d, \mathbf{l}, \phi)^{t}$ (where ${ }^{t}$ means for transposition), obeys a system of ordinary differential equations $[48,49]$, see Appendix. The two above definitions of $\boldsymbol{\xi}_{\Phi}$ and $\boldsymbol{\xi}_{F}$, referred to as $\Phi$ and $F$ representations, are suitable for treating different types of electrical boundary conditions on the surface. The quantities attributed to either of these representations are supplied below with the index $P$ meaning $P=\Phi$ or $P=F$.

The $8 \times 8$ transfer matrix relating the values of the amplitude vector $\boldsymbol{\xi}_{P}$ at the opposite edges of unit cell of a phononic crystal is a product of the transfer matrices $\hat{\mathbf{M}}_{P, i}=$ $\exp \left(i h_{i} \hat{\mathbf{N}}_{P, i}\right)$ through each individual $i$ th layer, where $h_{i}$ is its thickness and $\hat{\mathbf{N}}_{P, i}$ is the matrix of coefficients of Eq. (A6). Let the period of the phononic crystal be symmetric with respect to its middle plane (e.g., Fig. 1), which means that the unit cell consists of $2 n+1$ layers, the $i$ th and the $(2 n+2-i)$ th layer, $i=1, \ldots, n$, being of the same material and of the same thickness, so that $\hat{\mathbf{M}}_{P, i} \equiv \hat{\mathbf{M}}_{P, 2 n+1-i}$. As a result, the transfer matrix $\hat{\mathbf{M}}_{P}^{(S)}$ of a symmetric unit cell (the symbol ${ }^{S}$ refers to symmetry) takes the form

$$
\hat{\mathbf{M}}_{P}^{(S)}=\hat{\mathbf{M}}_{P, 1} \hat{\mathbf{M}}_{P, 2} \cdots \hat{\mathbf{M}}_{P, n+1} \cdots \hat{\mathbf{M}}_{P, 2} \hat{\mathbf{M}}_{P, 1} .
$$

Our subsequent considerations are based on the properties of the solutions of the eigenvalue problem

$$
\hat{\mathbf{M}}_{P}^{(S)} \zeta_{P, \alpha}=\gamma_{\alpha} \zeta_{P, \alpha}, \quad \alpha=1, \ldots, 8,
$$

which are discussed in Appendix B. For the future use, denote the eigenvector components as follows:

$$
\begin{aligned}
& \zeta_{\Phi, \alpha}=\left(\begin{array}{c}
\mathbf{U}_{\Phi, \alpha} \\
\mathbf{V}_{\Phi, \alpha}
\end{array}\right), \quad \mathbf{U}_{\Phi, \alpha}=\left(\begin{array}{c}
\mathbf{A}_{\alpha} \\
\Phi_{\alpha}
\end{array}\right), \quad \mathbf{V}_{\Phi, \alpha}=\left(\begin{array}{c}
\mathbf{L}_{\alpha} \\
D_{\alpha}
\end{array}\right), \\
& \zeta_{F, \alpha}=\left(\begin{array}{c}
\mathbf{U}_{F, \alpha} \\
\mathbf{V}_{F, \alpha}
\end{array}\right), \quad \mathbf{U}_{F, \alpha}=\left(\begin{array}{c}
\mathbf{A}_{\alpha} \\
D_{\alpha}
\end{array}\right), \quad \mathbf{V}_{F, \alpha}=\left(\begin{array}{c}
\mathbf{L}_{\alpha} \\
\Phi_{\alpha}
\end{array}\right) .
\end{aligned}
$$

Since the transfer matrix acts both on the eigenvectors $\zeta_{P, \alpha}$ and on the wave-amplitude vector $\boldsymbol{\xi}_{P}(y)$, the components $\mathbf{A}_{\alpha}, \Phi_{\alpha}, \mathbf{L}_{\alpha}$, and $D_{\alpha}$ of $\zeta_{P, \alpha}$ are related, respectively, to the mechanical displacement, electric potential, normal traction, and normal component of electric displacement, the two latter ones multiplied by $i$.

It should be noted that the eigenvalues of the transfer matrix through an asymmetric unit cell also occur in pairs (B3) or (B4) and this underlies the general partitioning of the plane $(\omega, k)$ in pass bands and stop bands, see, e.g., Ref. [47]. On the other hand, the transfer-matrix properties (B1), (B2) and the resulting relations (B6), (B7) $)_{1}$, and (B9) $)_{1}$ on the eigenvectors $\zeta_{P, \alpha}$ are not valid in the case of asymmetric unit cell and hold true specifically in the case of symmetric unit cell. It is this dissimilarity which underlies the difference in the analysis and in the results for SAWs in these two cases.

\section{EXISTENCE OF SAWS}

Aiming at the SAWs, we will focus our attention to the so-called full stop bands which are the spectral zones where all eight eigenvalues of $\hat{\mathbf{M}}_{P}^{(S)}$ appears in pairs (B4), namely, $\gamma_{\alpha}=1 / \gamma_{\alpha+4}^{*}, \quad\left|\gamma_{\alpha}\right| \neq 1, \quad \alpha=1, \ldots, 4$. Choosing $\left|\gamma_{\alpha}\right|<1$, $\alpha=1,2,3,4$, the wave-field amplitude $\xi_{P}(y)$ of the sought 
SAW taken at the surface $y=0$ of a half-space $y \geqslant 0$ may be represented as a linear combination

$$
\left.\boldsymbol{\xi}_{P}\right|_{y=0}=\sum_{\alpha=1}^{4} b_{\alpha} \zeta_{P, \alpha} \equiv\left(\begin{array}{c}
\mathbf{U}_{P} \\
\mathbf{V}_{P}
\end{array}\right),
$$

where the coefficients $b_{\alpha}$ are found from the mechanical and electrical boundary conditions at $y=0$. Each eigenvector $\zeta_{P, \alpha}$ of this superposition gives rise to a partial mode which decays with depth due to a factor $m \gamma_{\alpha}$ acquired after passing $m$ periods.

\section{A. Mechanically free electrically closed and electrically open surface}

Consider in parallel two types of boundary conditions at the surface $y=0$ of a given semi-infinite phononic crystal. The first is the condition of a mechanically free electrically closed surface, which implies the vanishing of the normal traction and electrical potential. According to Eq. (6), this condition can be formulated as

$$
\mathbf{V}_{F}=\sum_{\alpha=1}^{4} b_{\alpha} \mathbf{V}_{F, \alpha}=\mathbf{0} .
$$

The second is the condition of a mechanically free electrically open surface, which implies zero normal traction and zero normal component of electric displacement, that is, in view of Eq. (6),

$$
\mathbf{V}_{\Phi}=\sum_{\alpha=1}^{4} b_{\alpha} \mathbf{V}_{\Phi, \alpha}=\mathbf{0} .
$$

Physically, the former condition is realized on the surface coated with a thin metallic film. The latter condition is a somewhat model case which is, however, of clear interest for several reasons. First of all, it will be seen in Sec. III B that the information on the existence of SAWs on the electrically open surface, as well as on the electrically closed surface, is essentially involved in the existence considerations of SAWs on the crystal-vacuum interface. Second, this condition can be viewed as the limiting case of the boundary condition on a crystal-vacuum interface when the relative dielectric permittivity of the crystal is far greater than unity. Third, all considerations to be developed for the case of a piezoelectric phononic crystal with an electrically open surface remain valid in the case of a piezomagnetic phononic crystal with a thin superconductive coating merely via interpreting $\mathbf{D} \cdot \mathbf{n}$ as the normal component of magnetic induction, because the piezomagnetic effect is described by a thirdorder tensor with the same symmetry in indices as the piezoelectric tensor [50].

The immediate form of the dispersion equations corresponding to the boundary conditions (7) or (8) is $\operatorname{det} \hat{\mathbf{V}}_{P}=0$, but it is not suited for analyzing the SAW existence, in particular, since the matrices $\hat{\mathbf{V}}_{P}$ are not Hermitian. An equivalent form $\operatorname{det} \hat{\mathbf{Z}}_{P}=0$, where $\mathbf{Z}_{P}, P=F, \Phi$, are the Hermitian impedances of the half-infinite phononic crystal (B10)-(B12), is expedient for numerical computations but it is not suited for analyzing the SAW existence either, in particular because of the presence of poles disrupting monotonicity (B14), see the discussion in Sec. III D.
The admissible number of SAWs can be established via introducing the real symmetric matrices

$$
\hat{\mathbf{B}}_{P}=i \hat{\mathbf{V}}_{P} \hat{\mathbf{V}}_{P}^{t}
$$

equal to the product of matrices $\hat{\mathbf{V}}_{P}$ (B8) and analyzing the dispersion equation on the electrically closed or open surface written in the form

$$
\operatorname{det} \hat{\mathbf{B}}_{F}=0 \text { or } \operatorname{det} \hat{\mathbf{B}}_{\Phi}=0,
$$

respectively, since $\operatorname{det} \hat{\mathbf{B}}_{P}=\left(\operatorname{det} \hat{\mathbf{V}}_{P}\right)^{2}$. The required properties of $\hat{\mathbf{B}}_{P}$ are considered in Appendix C.

Properties (C10) and (C11) reveal that each of Eqs. (10) cannot have more than two roots in a full stop band. Hence,

for a fixed $k$ at most two SAWs per full stop band can exist on the mechanically free electrically closed surface.

(The reference to a fixed wave number $k$ and to the mechanically free conditions is skipped in the formulation of subsequent statements.)

From property (C10) it follows that the maximum number of SAWs permitted by the above statement may come about provided that all four eigenvalues of the matrices $\hat{\mathbf{B}}_{F}$ are positive near the lower edge $\omega_{l}$ of the full stop band $\omega_{l}<$ $\omega<\omega_{u}$. This may be the case in any full stop band; moreover, according to (C5), this is always so in the lowest stop band $0<\omega<\omega_{u}$.

The matrix $\hat{\mathbf{B}}_{\Phi}$, may also have all four eigenvalues positive at the lower edges of full stop bands, but with exception of the lowest one. According to Eq. (C6), $\hat{\mathbf{B}}_{\Phi}$ has three positive and one negative eigenvalue at $\omega=0$. Therefore, in view of properties (C7), (C8), and (C10),

only one SAWs in the lowest full stop band can exist on the electrically open surface;

at most two SAWs per upper full stop band can exist on the electrically open surface.

Here an "upper" stop band $\omega_{l}<\omega<\omega_{u}$ implies the one with $\omega_{l} \neq 0$.

In addition, properties (C12) and (C13) of the eigenvalues of the matrices $\hat{\mathbf{B}}_{P}$ allows us to conclude that

if two SAWs in a full stop band exist at $\omega_{F 1} \leqslant \omega_{F 2}$ on the electrically closed surface, then only one SAW exists on the electrically open surface and its frequency $\omega_{\Phi}$ is such that $\omega_{F 1} \leqslant \omega_{\Phi} \leqslant \omega_{F 2}$

and

if two SAWs in a full stop band exist at $\omega_{\Phi 1} \leqslant \omega_{\Phi 2}$ on the electrically open surface, then only one SAW exists on the electrically open surface and its frequency $\omega_{F}$ is such that $\omega_{\Phi 1} \leqslant \omega_{F} \leqslant \omega_{\Phi 2}$.

An existence criterion can be derived only for SAWs in the lowest stop band on the electrically closed surface. Bearing in mind (C5) and the discussion at the end of Appendix $\mathrm{C}$, we conclude that

at least one SAW in the lowest stop band must exist on the electrically closed surface unless the limiting wave at $\omega=\omega_{u}$ satisfies the boundary condition at this surface. 
Since the limiting wave usually does not satisfy the boundary condition, one SAW usually does exist on the electrically closed surface in the lowest stop band. A similar criterion cannot be extended to SAWs in the lowest stop band on the electrically open surface or to SAWs in the upper stop bands on any of two types of surface, because none of these cases guarantees positiveness of all four eigenvalues of $\hat{\mathbf{B}}_{P}$ at the stop band lower edge.

\section{B. Phononic crystal-vacuum interface}

The phononic crystal-vacuum interface $y=0$, otherwise termed the mechanically and electrically free surface, requires the traction to vanish and the electric potential together with the normal component of electric displacement to be continuous with those in the vacuum $(y<0)$, where the wave field (1) is accompanied by a wave of electric potential $\varphi^{(v)}(\mathbf{r}, t)=$ $\Phi^{(v)} e^{k y+i(k x-\omega t)}(k>0)$. Thus the corresponding boundary condition can be written in terms of (6) and (5) as

$$
\sum_{\alpha=1}^{4} b_{\alpha} \mathbf{L}_{\alpha}=0, \quad \sum_{\alpha=1}^{4} b_{\alpha} \Phi_{\alpha}=\Phi^{(v)}, \quad \sum_{\alpha=1}^{4} b_{\alpha} D_{\alpha}=-i k \varepsilon_{0} \Phi^{(v)}
$$

where $\varepsilon_{0}$ is the dielectric permittivity of vacuum. Using the admittance definition (B11) 2 and the mechanical boundary condition (11) yields the equality $D=i Y_{F, 44} \Phi$, where $\Phi=\sum_{\alpha=1}^{4} b_{\alpha} \Phi_{\alpha}, D=\sum_{\alpha=1}^{4} b_{\alpha} D_{\alpha}$ and $Y_{F, 44}$ is the 44th element of matrix $\hat{\mathbf{Y}}_{F}$ defined by (B10) $)_{2}$. Hence, in view of the electrical boundary conditions $(11)_{2,3}$, the SAW dispersion equation can be written in the form

$$
Y_{F, 44}=-k \varepsilon_{0} \text {. }
$$

The roots of Eq. (12) in a full stop band $\omega_{l}<\omega<\omega_{u}$ may be analyzed graphically, taking advantage of the properties of $Y_{F, 44}$ as a function of $\omega$ at a fixed $k$. In particular, according to Eqs. (B14) and (B15), $\partial Y_{F, 44} / \partial \omega$ is positive and $Y_{F, 44}>$ 0 at $\omega=0$. Furthermore, since formally setting $\varepsilon_{0}=0$ or $\varepsilon_{0}=\infty$ reduces Eq. (11) to the boundary conditions on the mechanically free electrically open or electrically closed surface, respectively, the same replacement made in (12) shows that $Y_{F, 44}$ vanishes at the frequencies $\omega_{\Phi i}$ of SAWs on the electrically open surface and has poles at the frequencies $\omega_{F i}$ of SAWs on the electrically closed surface. Note that $Y_{F, 44}$ is normally finite at the edge frequencies $\omega_{l}, \omega_{u}$, at which it diverges only in the exceptional case when the limiting wave happens to satisfy the boundary condition of the mechanically free electrically closed surface.

Possible types of behavior of the function $Y_{F, 44}(\omega)$ are depicted in Figs. 2 and 3. On examining these figures with Eq. (12) borne in mind, the overall conclusion is that

at most two SAWs per full stopband can exist on the electrically free surface.

On top of this statement, Figs. 2 and 3 link the number of SAWs on the mechanically and electrically free surface to that on the electrically closed and open surface. In particular, Fig. 2 provides the following conclusions regarding SAWs in the lowest stop band. At least one SAW exists on the electrically free surface if two SAWs exist on the electrically closed surface [Figs. 2(a) and 2(b)]. At most one SAW exists
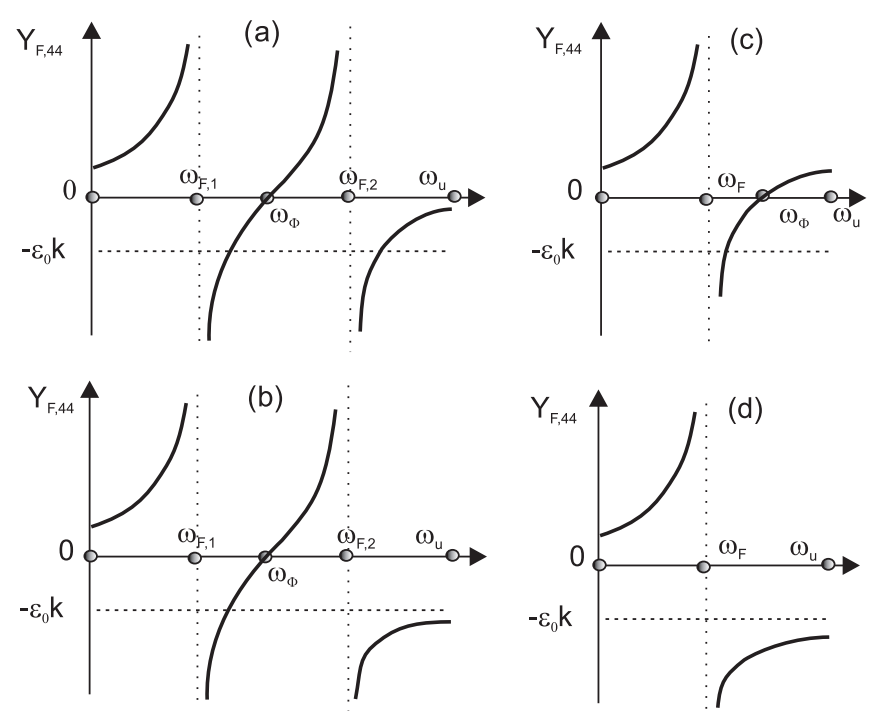

FIG. 2. Possible options for the shape of the function $Y_{F, 44}(\omega)$ in the lowest stop band $0<\omega<\omega_{u}$.

on the electrically free surface if only one SAWs exists on the electrically closed surface [Figs. 2(c) and 2(d)]. At least one SAW must exist on the electrically free surface if the SAW exists on the electrically open surface [Fig. 2(c)]. Note that these three statements apply as well to such upper stop bands $\omega_{l}<\omega<\omega_{u}$, where $Y_{F, 44}$ at $\omega_{l} \neq 0$ is greater than $-k \varepsilon_{0}$.

Additional possibilities are allowed in those upper stop bands where $Y_{F, 44}\left(\omega_{l}\right)<-k \varepsilon_{0}$. In this case, two SAWs may exist on the electrically free surface even if only one SAW exists on the electrically closed surface [Fig. 3(a)]. One SAW may exist on the electrically free surface even if no SAW exists on both electrically closed and open surfaces [Figs. 3(b) and 3(c)]. At least one SAW must exist on the electrically free surface if two SAWs exist on the electrically open surface [Fig. 3(d)]. The latter statement takes
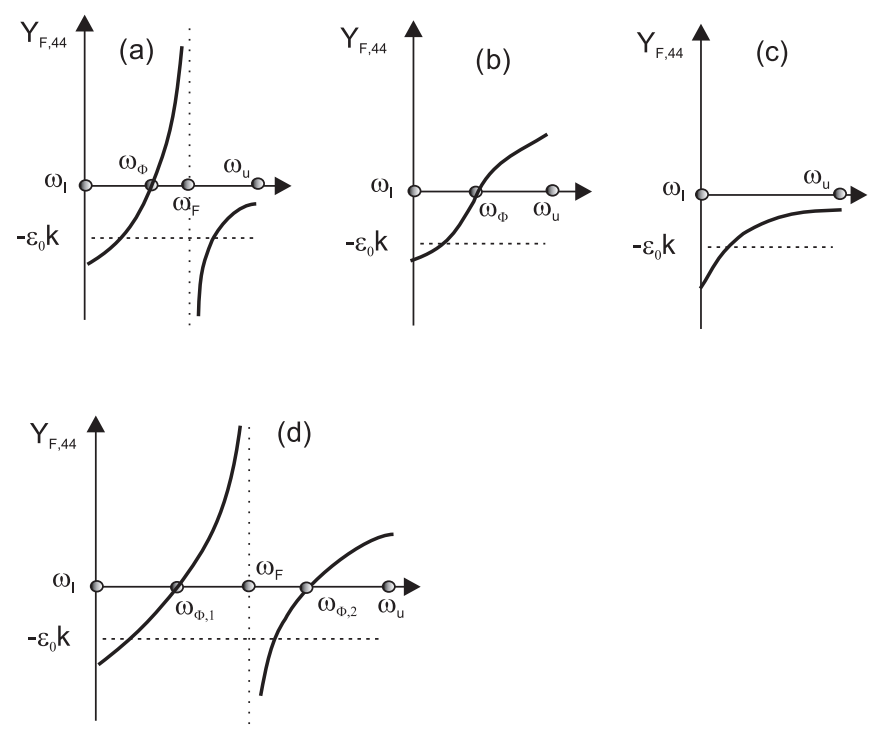

FIG. 3. Possible options for the shape of the function $Y_{F, 44}(\omega)$ when $Y_{F, 44}\left(\omega_{l}\right)<-k \varepsilon_{0}$. 
into account property (C5) and it holds regardless of the inequality between $Y_{F, 44}\left(\omega_{l}\right)$ and $-k \varepsilon_{0}$; however, the sign of this inequality decides the existence of the second SAW on the electrically open surface, see Fig. 3(d).

Note that the boundary condition (11) substituted into the impedance definition (B10) leads to the dispersion equation in the form $\operatorname{det} \hat{\mathbf{Z}}_{F}+\frac{1}{k \varepsilon_{0}} \operatorname{det} \hat{\mathbf{Z}}_{F}^{3 \times 3}=0$ where $\hat{\mathbf{Z}}_{F}^{3 \times 3}$ is the $3 \times 3$ upper diagonal block of $\hat{\mathbf{Z}}_{F}$ (B10) . This is equivalent to (12) in view of the identity $Y_{F, 44}=\operatorname{det} \hat{\mathbf{Z}}_{F}^{3 \times 3} / \operatorname{det} \hat{\mathbf{Z}}_{F}$, unless a coincidentally simultaneous vanishing of $\operatorname{det} \hat{\mathbf{Z}}_{F}$ and $\operatorname{det} \hat{\mathbf{Z}}_{F}^{3 \times 3}$ at some common frequency and wave number $\left(\omega_{0}, k_{0}\right)$. Such solution to (11) fails to be detected by Eq. (12). The condition $\operatorname{det} \hat{\mathbf{Z}}_{F}^{3 \times 3}=0$ is equivalent to $\operatorname{det} \hat{\mathbf{Z}}_{\Phi}=0$, since both imply existence of the vector $\left(\mathbf{U}_{P}, \mathbf{V}_{P}\right)^{t}$ with $\mathbf{L}=\mathbf{0}, D=0$. Thus $\left(\omega_{0}, k_{0}\right)$ is an exceptionally possible common root of the dispersion equations $\operatorname{det} \hat{\mathbf{Z}}_{F}=0$ and $\operatorname{det} \hat{\mathbf{Z}}_{\Phi}=0$ corresponding to the electrically closed and electrically open boundary conditions, respectively (see Sec. III A). This solution describes one of the two following options. First, if $\hat{\mathbf{Z}}_{F}$ and $\hat{\mathbf{Z}}_{\Phi}$ have the null vectors $\mathbf{U}_{F}=(\mathbf{A}, D)^{t}$ and $\mathbf{U}_{\Phi}=(\tilde{\mathbf{A}}, \Phi)^{t}$ fulfilling $\hat{\mathbf{Z}}_{P} \mathbf{U}_{P}=\mathbf{0}$ with different mechanical displacements $\mathbf{A}$ and $\tilde{\mathbf{A}}$, then two independent SAWs with $\mathbf{V}_{F}=\mathbf{0}$ and $\mathbf{V}_{\Phi}=\mathbf{0}$, respectively, occur at $\left(\omega_{0}, k_{0}\right)$. Their linear combination can always be arranged so that it satisfies the boundary condition (11). Second, if $\hat{\mathbf{Z}}_{F}$ and $\hat{\mathbf{Z}}_{\Phi}$ happen to have the null vectors with the same mechanical displacements, then $\mathbf{U}_{F}=(\mathbf{A}, 0)^{t}$ and $\mathbf{U}_{\Phi}=(\mathbf{A}, 0)^{t}$ and a single SAW occurs at $\left(\omega_{0}, k_{0}\right)$ which produces neither electric potential nor electric displacement on the surface ( $\Phi=0$ and $D=0$ at $y=0)$ and hence does not excite an electric wave in the vacuum, thus satisfying (11) with $\Phi^{(v)}=0$ and $D^{(v)}=0$.

It is seen that if the above specific solution $\left(\omega_{0}, k_{0}\right)$ occurs, it eliminates one zero and one pole of the function $Y_{F, 44}(\omega)$ out of their total number admissible per a full stop band. In other words, by (B14) $\operatorname{det} \hat{\mathbf{Z}}_{F}=F(\omega)\left(\omega-\omega_{0}\right)$ and $\operatorname{det} \hat{\mathbf{Z}}_{F}^{3 \times 3}=f(\omega)\left(\omega-\omega_{0}\right)$ for a fixed $k=k_{0}$, and, in view of the interrelation between the existence of SAWs on the electrically closed and open surfaces (Sec. III A), either none of the functions $F(\omega)$ and $f(\omega)$ vanishes in the given stop band or only one of them vanishes once. As a result, the function $Y_{F, 44}(\omega)=f(\omega) / F(\omega)$ either keeps permanent sign or else it has either one pole or one zero within the stop band. It is graphically evident that under these conditions (12) can have at most one root on top of the assumed solution $\left(\omega_{0}, k_{0}\right)$. Thus the general statement regarding the maximum number of SAWs on the electrically free surface remains valid in this special case.

\section{Phononic crystals with crystallographic symmetry}

Assume that all layers constituting a given phononic crystal have symmetry planes parallel to a common plane orthogonal to their interfaces. Let the sagittal plane spanned by the vectors $\mathbf{m}$ and $\mathbf{n}$ coincide with this plane. Then system (A6) splits into six and two decoupled equations describing six piezoactive sagittally polarized modes ( $\mathrm{S}$ modes) and two nonpiezoactive horizontally polarized modes (SH modes). Correspondingly, the transfer matrix splits into the $6 \times 6$ and $2 \times 2$ diagonal blocks. The S-SAW involves three partial modes and the occurrence of SAWs on a mechanically free surface is determined by the $3 \times 3$ submatrices $\hat{\mathbf{B}}_{P}^{3 \times 3}$ obtained from the $4 \times 4$ matrices $\hat{\mathbf{B}}_{P}$ by deleting third row and third column. A diagonal submatrix retains the sign-definiteness features of the main matrix; hence, by (C4)-(C6), the eigenvalues of $\hat{\mathbf{B}}_{P}^{3 \times 3}$ are decreasing functions of $\omega$ and there are three positive eigenvalues of $\hat{\mathbf{B}}_{F}^{3 \times 3}$ and two positive ones of $\hat{\mathbf{B}}_{\Phi}^{3 \times 3}$ at $\omega=0$. As a result, the principal conclusions obtained for the general case modify with regarding S-SAWs as follows:

only one S-SAW per a full stop band can exist on the electrically closed or open surface;

only one S-SAW in the lowest stop band and at most two SSAW per an upper full stop band can exist on the electrically free surface.

Now let the layers share an even-fold symmetry axis lying in the interfacial plane and let the sagittal plane be orthogonal to this axis. In this case, there are two decoupled sets of four nonpiezoactive $\mathrm{S}$-modes and four piezoactive $\mathrm{SH}$-modes. The latter are described by the lowest $2 \times 2$ diagonal blocks $\hat{\mathbf{B}}_{P}^{2 \times 2}$ of $\hat{\mathbf{B}}_{P}$. Note that one of the eigenvalue of $\hat{\mathbf{B}}_{\Phi}$, which is negative at $\omega=0$ by Eq. (C6) is the one associated with piezoactive modes, i.e., with $\hat{\mathbf{B}}_{\Phi}^{2 \times 2}$ in the present case. Besides, statement (C7) adapted for the present case implies that the blocks $\hat{\mathbf{B}}_{\Phi}^{2 \times 2}$ and $\hat{\mathbf{B}}_{F}^{2 \times 2}$ cannot have all four eigenvalues of the same sign. Hence, first, there are two positive eigenvalues of $\hat{\mathbf{B}}_{F}^{2 \times 2}$ and one positive eigenvalue of $\hat{\mathbf{B}}_{\Phi}^{2 \times 2}$ at $\omega=0$. Second, given $\omega=\omega_{l} \neq 0$, if two eigenvalues of $\hat{\mathbf{B}}_{\Phi}^{2 \times 2}$ are positive, then the two eigenvalues of $\hat{\mathbf{B}}_{F}^{2 \times 2}$ cannot be both positive and vice versa. Therefore

no SH-SAW exists in the lowest stop band and only one SH-SAW can exist per full stop band on the electrically open surface;

only one SH-SAW per upper full stop band can exist on the electrically closed or electrically free surface;

if SH-SAW exists on the electrically closed surface, then no SH-SAW in the same stop band exists on the electrically open surface, and vice versa.

It should be added that decoupling of S and $\mathrm{SH}$ modes implies an independent definition of the respective spectral bands, so that a full stop band of $\mathrm{S}$ modes can overlap a pass band of SH-modes and, vice versa, a stop band of SH modes can overlap a pass band of S-modes. In the case of stop/pass band overlap, an arbitrary perturbation breaking the sagittalplane symmetry transforms S-SAW or SH-SAW into a leaky wave. By contrast, a SAW existing in a pass band and being subjected to a certain particular perturbation may remain nonleaky despite polarization mixing, see Refs. [32,51]. In the case of overlapping stop bands, the numbers of S- and SH-SAWs can predetermine in a certain way the properties of SAWs of general polarization arising under perturbation of crystallographic symmetry. Consider an example of a full stop band of piezoactive S-SAWs $\omega_{l}^{S}<\omega<\omega_{u}^{S}$, which overlaps with a full stop band of SH-SAWs $\omega_{l}^{\mathrm{SH}}<\omega<\omega_{u}^{\mathrm{SH}}$. Recall that SH modes are nonpiezoactive once S-SAWs are 
piezoactive. According to Ref. [46], no nonpiezoactive SHSAWs can exist on the mechanically free surface of the phononic crystal with symmetric unit cell, whereas the limiting bulk SH mode either at $\omega_{l}^{\mathrm{SH}}$ or at $\omega_{u}^{\mathrm{SH}}$ is exceptional, that is, it satisfies the traction-free boundary condition. Assume that the latter exceptional SH wave is at $\omega_{u}^{\mathrm{SH}}$ and that $\omega_{u}^{\mathrm{SH}}<$ $\omega_{u}^{S}$. In addition, let two S-SAWs exist on the electrically free surface in the common frequency interval $\max \left(\omega_{l}^{\mathrm{SH}}, \omega_{l}^{S}\right)<$ $\omega<\omega_{u}^{\mathrm{SH}}$, as it is permitted provided that the stop band is not the lowest one. Under a weak perturbation breaking the symmetric orientation of the sagittal plane (such as its deviation or a change of material constants), the two S-SAWs merely acquire an out-of-plane polarization component while remaining near-surface localized. It can be thought that such perturbation, at least some special one, can lead to the localization of the formerly bulk exceptional SH mode. However, the appearance of a third SAW is excluded, and so we arrive at a somewhat counterintuitive conclusion that a weak perturbation in the above case cannot transform the exceptional $\mathrm{SH}$ mode to a SAW. On the other hand, if the overlapping stop bands are the lowest ones and one S-SAWs comes about in the common interval $0<\omega<\omega_{u}^{\mathrm{SH}}$, then a perturbation breaking the symmetric configuration may transform the exceptional $\mathrm{SH}$ mode at $\omega_{u}^{\mathrm{SH}}$ to the SAW with quasi-SH polarization, since the latter event does not contradict the statement concerning the number of SAWs in the lowest stop bands.

\section{Mechanically clamped surface}

The condition of vanishing mechanical displacement $\mathbf{a}=\mathbf{0}$ at the surface $y=0$ (a clamped surface) is of relatively minor importance as only a limiting model for a contact with an idealized absolutely rigid exterior body. At the same time, the existence or nonexistence of SAWs on a "fictitiously" clamped surface decides about the presence or absence of the poles of the determinants of the impedances $\hat{\mathbf{Z}}_{P}$. This fact is important for the analysis of the SAW propagation, e.g., on a loaded surface of a phononic crystal or on an interface between two phononic crystals.

The boundary conditions on the mechanically clamped electrically closed or electrically open surface is $\mathbf{U}_{P}=$ $\sum_{\alpha=1}^{4} b_{\alpha} \mathbf{U}_{P, \alpha}=\mathbf{0}$. By the similar reasons as outlined above Eq. (10), the SAW existence in this case is expedient to analyze in terms of the matrices

$$
\hat{\mathbf{Q}}_{P}=i \hat{\mathbf{U}}_{P} \hat{\mathbf{U}}_{P}^{t}
$$

where the matrices $\hat{\mathbf{U}}_{P}$ are introduced in Eq. (B8). The corresponding form of the dispersion equation is

$$
\operatorname{det} \hat{\mathbf{Q}}_{F}=0 \text { or } \operatorname{det} \hat{\mathbf{Q}}_{\Phi}=0
$$

on the mechanically clamped electrically closed or electrically open surface, respectively. In view of the properties (C14) of $\hat{\mathbf{Q}}_{P}$, we conclude that

no SAW exists in the lowest full stop band on the mechanically clamped surface;

at most two SAWs per upper full stop band exist on the clamped electrically closed or open surface.

Thus the determinants of $\hat{\mathbf{Z}}_{P}$ have no poles in the lowest stop band and at most two poles each in the upper stop bands.

\section{E. SAWs at $k=0$}

Propagation of acoustoelectric waves with a small tangential wave number $k$, i.e., along the directions which are close to the normal $\mathbf{n}$ to the layer interfaces, generally cannot be described with the aid of the quasielectrostatic approximation which ceases to be applicable once $k$ becomes small enough to be commensurate with $\omega / c$, where $c$ is the electromagnetic wave velocity. For such $k$, the two modes of the quasielectrostatic potential coupled with mechanical displacement via the piezoelectric effect transform into a pair of electromagnetic waves. Two more electromagnetic waves arise from two modes of the quasistatic magnetic potential, which are ignored under the quasielectrostatic approximation due to their decoupling from the acoustoelectric fields (unless the material possesses piezomagnetic and/or magnetoelectric properties). This opens up a particular mechanism of phonon-polariton coupling [52-55]. Altogether, it follows that a correct tracing of the limiting transition $k \rightarrow 0$ requires a full treatment of the electromechanical waves such that implies a replacement of the present $8 \times 8$ formalism with the $10 \times 10$ one incorporating six acoustic partial modes coupled with four electromagnetic modes [56-58]. We shall not, however, pursue here this formalism and consider a zero value $k=0$ as a secluded point of the dispersion dependence. In this context, the electromagnetic waves propagating along the same direction $\mathbf{n}$ with far higher speed then the acoustoelectric ones may be disregarded and the quasielectrostatic approximation can be used.

From the explicit form (A7) of $\hat{\mathbf{N}}_{\Phi}$, it follows that at $k=0$ this matrix has a doubly degenerate zero eigenvalue and becomes nonsemisimple (nondiagonalizable, see, e.g., Ref. [59]), that is, instead of two linearly independent eigenvectors, $\hat{\mathbf{N}}_{\Phi}$ has one eigenvector $\boldsymbol{\xi}_{\Phi, d}$ and one so-called generalized eigenvector $\boldsymbol{\xi}_{\Phi, g}$ which fulfill the relations $\hat{\mathbf{N}}_{\Phi} \boldsymbol{\xi}_{\Phi, d}=$ $\mathbf{0}$ and $\hat{\mathbf{N}}_{\Phi} \boldsymbol{\xi}_{\Phi, g}=-\boldsymbol{\xi}_{\Phi, d}$. The nonzero components of these vectors are $\Phi_{d}$ in $\boldsymbol{\xi}_{\Phi, d}$ and $\mathbf{V}_{\Phi, g} \equiv\left(\mathbf{L}_{g}, D_{g}\right)^{t}=(n n)\left(\mathbf{0}, \Phi_{d}\right)^{t}$ in $\boldsymbol{\xi}_{\Phi, g}$. The corresponding partial solutions to Eq. (A6) in a homogeneous layer, numbered by $\alpha=7$ and 8 , are $\boldsymbol{\xi}_{\Phi, 7}(y)=$ $\boldsymbol{\xi}_{\Phi, d}$ and $\boldsymbol{\xi}_{\Phi, 8}(y)=\boldsymbol{\xi}_{\Phi, g}-i y \boldsymbol{\xi}_{\Phi, d}$.

Since the eighth line of $\hat{\mathbf{N}}_{\Phi}$ at $k=0$ is identically zero, the other six partial solutions $\boldsymbol{\xi}_{\Phi, \alpha}(y), \alpha=1, \ldots, 6$, of (A6) associated with nonvanishing eigenvalues of $\hat{\mathbf{N}}_{\Phi}$ have zero eighth component which is the normal component of electric displacement $\mathbf{D}_{\alpha} \cdot \mathbf{n}$. This is consistent with the fact that these six partial solutions describe bulk waves propagating along $\mathbf{n}$ and hence the components of their electric displacement along $\mathbf{n}$ must be equal to zero. It thus follows that the solution $\boldsymbol{\xi}_{\Phi, 8}(y)$ with a constant electric-displacement component $D_{g}$ should be discarded. The six partial modes $\boldsymbol{\xi}_{\alpha}(y)=\left(\mathbf{a}_{\alpha}, \phi_{\alpha}, \mathbf{l}_{\alpha}, 0\right)^{t}, \alpha=$ $1, \ldots, 6$, are just enough to satisfy the mechanical boundary condition at the surface and the continuity conditions at the layer interfaces. The continuity of electric potential at the layer interfaces and at the crystal surface can always be ensured by way of adding an appropriate piecewise constant value of $\boldsymbol{\xi}_{\Phi, 7}(y)$, which at the same time does not affect the mechanical boundary conditions.

On this basis, we conclude that the normal propagation of acoustoelectric waves in a phononic crystal can be described by the $6 \times 6$ transfer matrix obtained from the $8 \times 8$ matrix 
$\hat{\mathbf{M}}_{\Phi}^{(S)}$ with $k=0$ by deleting the fourth and eighth lines and columns. The resulting matrix satisfies the same symmetry properties (B1) and (B2) with the appropriately redefined $6 \times 6$ matrix $\hat{\mathbf{T}}$. Correspondingly, the eigenvectors $\left(\mathbf{A}_{\alpha}, \mathbf{L}_{\alpha}\right)^{t}$, $\alpha=1, \ldots, 6$, of this transfer matrix allow one to construct the $3 \times 3$ impedance and admittance matrices, which are Hermitian and possess the same sign-definiteness properties (B14) and (B15) in the full stop bands. In consequence, an admissible number of normally propagating SAWs does not depend on the type of electrical boundary conditions at the surface and is determined by the same propositions as formulated in Ref. [46] for purely elastic 1D phononic crystals. In particular,

at $k=0$, only one SAW per full stop band can exist on the mechanically free surface of a piezoelectric phononic crystal with symmetric unit cell.

The above reasonings also apply to the phononic crystal with asymmetric unit cell, so, in accordance with Ref. [46],

at $k=0$, at most three SAWs per full stop band can exist on the mechanically free surface of a piezoelectric phononic crystal with asymmetric unit cell.

If either the normal to the layer interfaces or the normal to the sagittal plane is orthogonal to a common axis of crystallographic symmetry of layers, then one pair of partial acoustic modes $\boldsymbol{\xi}_{P, \alpha}(y)$ and $\boldsymbol{\xi}_{P, \alpha+3}(y)$ which are polarized along this axis uncouples from four others. One of these two modes, e.g., $\alpha$, is a candidate for a one-partial SAW in a stop band. It occurs that such a one-partial SAW cannot occur if the unit cell is symmetric. Indeed, had the mode $\alpha$ satisfied the traction-free boundary condition, it would be that the part $\mathbf{V}_{P, \alpha}$ of the eigenvector $\zeta_{P, \alpha}$ of $\hat{\mathbf{M}}^{(S)}$ vanishes but this is impossible in stop bands due to orthogonality condition (B7). However, if the unit cell is asymmetric, then one-partial SAW can exist, since the properties of the transfer matrix of asymmetric unit cell allow an eigenvector $\zeta_{P, \alpha}$ to have either $\mathbf{U}_{P, \alpha}=0$ or $\mathbf{V}_{P, \alpha}=0$ in stop bands.

\section{NUMERICAL EXAMPLE}

Our foregoing analysis has established on general grounds that at most two SAWs may exist in a given full stop band. This section demonstrates that the maximum possible number can actually be attained in a phononic crystal.

We shall compute SAW dispersion curves in the bilayered phononic crystal composed of alternating layers of ceramics PZT6B and of single-crystal $\mathrm{BaTiO}_{3}$. The material constants of PZT6B (symmetry $6 \mathrm{~mm}$ ) are $c_{11}^{E}=168, c_{12}^{E}=$ $84.7, c_{13}^{E}=84.2, c_{33}^{E}=163, c_{44}^{E}=35.5, c_{66}^{E}=41.65$ (all in GPa units); $e_{31}=-0.9, e_{33}=7.1, e_{15}=4.6$ (all in $\mathrm{C} / \mathrm{m}^{2}$ units); $\varepsilon_{11}^{S} / \varepsilon_{0}=407, \varepsilon_{33}^{S} / \varepsilon_{0}=386, \rho=7550 \mathrm{~kg} / \mathrm{m}^{3}$ [60]. The material constants of $\mathrm{BaTiO}_{3}$ (symmetry $4 \mathrm{~mm}$ ) are $c_{11}^{E}=$ $275, c_{12}^{E}=179, c_{13}^{E}=152, c_{33}^{E}=165, c_{44}^{E}=54.3, c_{66}^{E}=113$ (all in GPa units); $e_{31}=-2.65, e_{33}=3.64, e_{15}=21.3$ (all in $\mathrm{C} / \mathrm{m}^{2}$ units); $\varepsilon_{11}^{S} / \varepsilon_{0}=1744, \varepsilon_{33}^{S} / \varepsilon_{0}=97, \rho=6020 \mathrm{~kg} / \mathrm{m}^{3}$ [60]. The layers are oriented so that their crystallographic axes $X, Y, Z$, where $Z$ is parallel to the principal symmetry axis, are mutually aligned and the layer interface is the plane of symmetry [010]. Hence the propagation along the $X$ axis en-

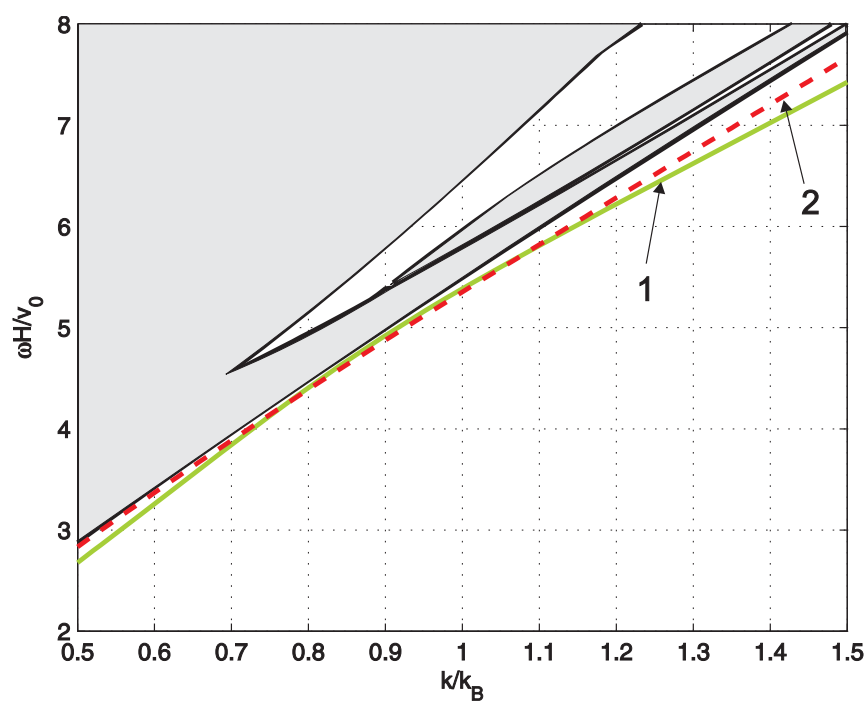

FIG. 4. Frequency vs wave number dispersion curves of the S-SAW and SH-SAW in the lowest stop band of PZT6B/BaTiO phononic crystal with the electrically closed surface. The propagation direction is parallel to the $X$ axis. White and grey areas are stop and pass bands, respectively. Curve 1: S-SAW, curve 2: SH-SAW. The velocity $v_{0}=3000 \mathrm{~m} / \mathrm{s}, H$ is the period, $k_{B}=2 \pi / H$ is the Brillouin wave number.

sures decoupling of nonpiezoactive $\mathrm{S}$ modes and piezoactive SH modes. Unless otherwise specified (see Figs. 7 and 8), the exterior layer is made of PZT6B and its thickness is $h$ while the thicknesses of the interior PZT6B and of the $\mathrm{BaTiO}_{3}$ layers are $2 h$ and $h$, respectively, so that the unit cell with a period $H=3 h$ counted from the surface is symmetric.

Figure 4 shows that the lowest stop band contains two frequency vs wave number dispersion curves of SAWs propagating on the electrically closed surface in the direction of the $X$ axis. One of the curves describes the nonpiezoactive S-SAW, the other the piezoactive SH-SAW. The latter exists solely due to the piezoelectric effect, since SH-SAW cannot occur in a purely elastic phononic crystals with symmetric period [46]. For clarity, the phase velocities of these S-SAW and SH-SAW are depicted in Fig. 5. Note that the are two values of the wave number, $k / k_{B} \approx 0.75$ and $\approx 1.075$, at which the frequencies of the two SAWs become equal. This event may be seen as an analog of the acoustic axes for bulk waves in anisotropic solids.

According to the results of Sec. III A, only the nonpiezoactive S-SAW but no piezoactive SH-SAWs can exist in the lowest stop band of a phononic crystal with symmetric unit cell if its mechanically free surface is electrically open. If the surface is electrically free, i.e. bounded by vacuum, then the frequency $\omega_{\mathrm{SH}}$ of piezoactive SH-SAW breaks off from the upper edge $\omega_{u}$ of the stop band (we note that in the phononic crystal under consideration $\omega_{u}$ confining the lowest stop-band common for $\mathrm{S}$ and $\mathrm{SH}$ modes is the edge frequency for $\mathrm{SH}$ modes). However, the appearing gap is extremely small due to very high dielectric permittivities of PZT6B and $\mathrm{BaTiO}_{3}$. On the other hand, the condition of electrically free boundary can be generalized by assuming that a given medium is bounded by a dielectric without a mechanical contact. Then the 


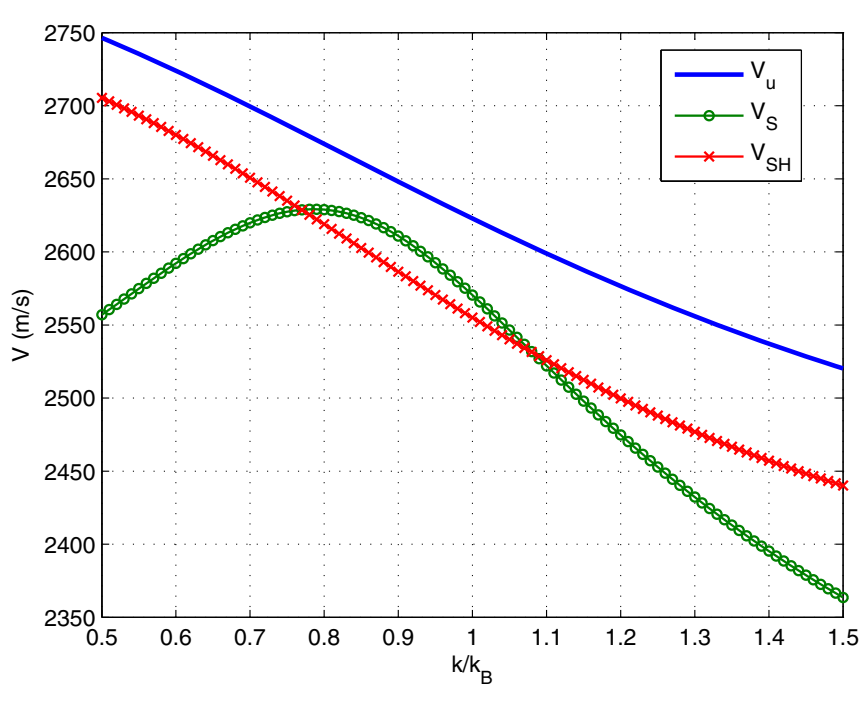

FIG. 5. Phase velocity vs wave number dispersion curves of the S-SAW and SH-SAW recalculated from the data of Fig. 4. $V_{S}$ is SSAW velocity, $V_{\mathrm{SH}}$ is SH-SAW velocity, $V_{u}=\omega_{u} / k$ is the limiting wave velocity associated with the upper edge of the lowest stop band.

dielectric permittivity of vacuum $\varepsilon_{0}$ should be replaced with the permittivity $\varepsilon$ of the dielectric exterior. Within this model, if the relative permittivity of the exterior is, for instance, taken to be 100 , then the SH-SAW frequency $\omega_{\mathrm{SH}}$ changes from $0.9987 \omega_{u}$ to $0.9981 \omega_{u}$ with $k / k_{B}$ changing from 0.5 to 1.5 , i.e., the gap becomes clearly seen.

Consider the propagation direction which slightly deviates from the $X$ axis. Then the two SAW branches should still exist, but now both SAWs are going to be piezoactive and of general polarization. Figure 6 shows the velocity versus wave-number dependence for the quasi-S-SAW and quasi-SH-SAW on the electrically closed surface in the direction making $10^{\circ}$ with the

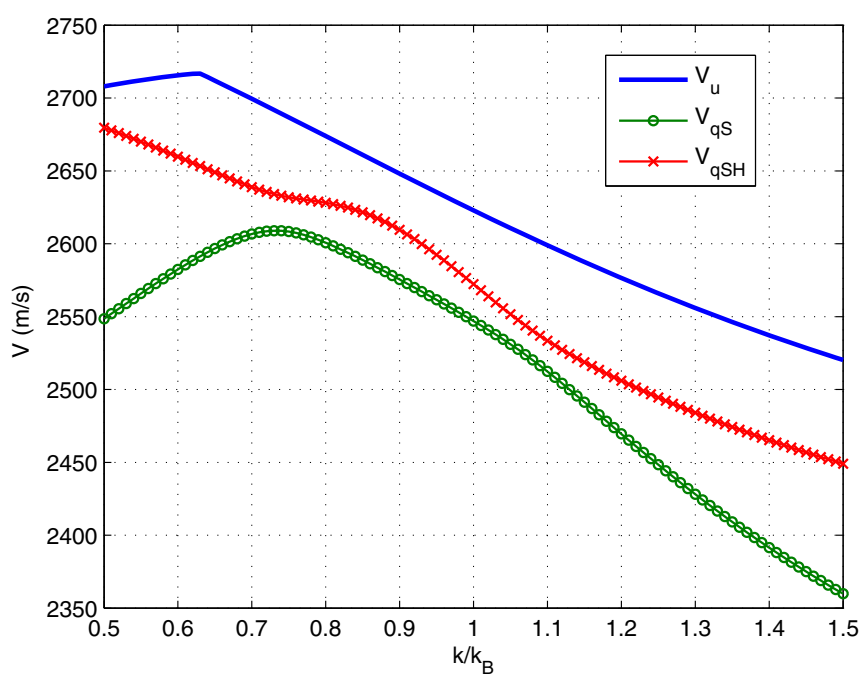

FIG. 6. Phase velocity vs wave number dispersion curves of the quasi-S-SAW and SH-SAW in the direction making $10^{\circ}$ with the $X$ axis. $V_{\mathrm{qS}}$ is quasi-S-SAW velocity, $V_{\mathrm{qSH}}$ is quasi-SH-SAW velocity, $V_{u}=\omega_{u} / k$ is the limiting wave velocity associated with the upper edge of the lowest stop band.
$X$ axis. Thus we observe an example of two piezoactive SAWs which is their maximum number possible in a full stop band. Comparing with Fig. 5, it is seen that the frequency (velocity) degeneracy of the two SAWs has been lifted. The results for the other types of electrical boundary conditions are also in agreement with the general predictions of Sec. III regarding the lowest stop band, namely, there is one quasi-S-SAW on the electrically open surface and two SAWs on the electrically free surface. It is noted that, due to a slight deviation from the $X$ axis, the quasi-S-SAW is only weakly piezoactive, so that its velocity on the electrically open and free surface differs very little from that on the electrically closed surface.

Consider another assemblage of the given layers, such that also has a symmetric unit cell but the exterior layer is made of $\mathrm{BaTiO} 3$ (with a thickness $0.5 h$, half of that of interior $\mathrm{BaTiO}_{3}$ layers). Denote this phononic crystal as $\mathrm{BaTiO}_{3} / \mathrm{PZT} 6 \mathrm{~B}$, to distinguish it from the $\mathrm{PZT} 6 \mathrm{~B} / \mathrm{BaTiO}_{3}$ structure with PZT6B exterior layer. Sticking to the lowest stop band and the $X$ axis propagation direction along the electrically closed surface, compare the SAW dispersion branches obtained for $\mathrm{BaTiO}_{3} / \mathrm{PZT} 6 \mathrm{~B}$ (Fig. 7) with those obtained previously for PZT6B $/ \mathrm{BaTiO}_{3}$ (Fig. 5). Both S-SAW and SH-SAW are seen to exist in either of these cases, but the shape of their branches markedly differs. It is of interest to trace the transition from one set of curves to the other as $\mathrm{PZT} 6 \mathrm{~B} / \mathrm{BaTiO}_{3}$ structure is being transformed to $\mathrm{BaTiO}_{3} / \mathrm{PZT} 6 \mathrm{~B}$ by way of changing the thickness of the exterior layer. Note that the so modelled intermediate configurations will have an asymmetric unit cell, and hence the maximum possible number of SAWs per a stop band in there may be less than in a structure with symmetric unit cell (see Ref. [47]). The SAW dispersion branches in PZT6B/BaTiO 3 with the exterior PZT6B layer of thickness $0.9 \mathrm{~h}$ are shown as curves 1 and 2 in Fig. 8. It is seen that there appears an interval of $k$ values, within which the

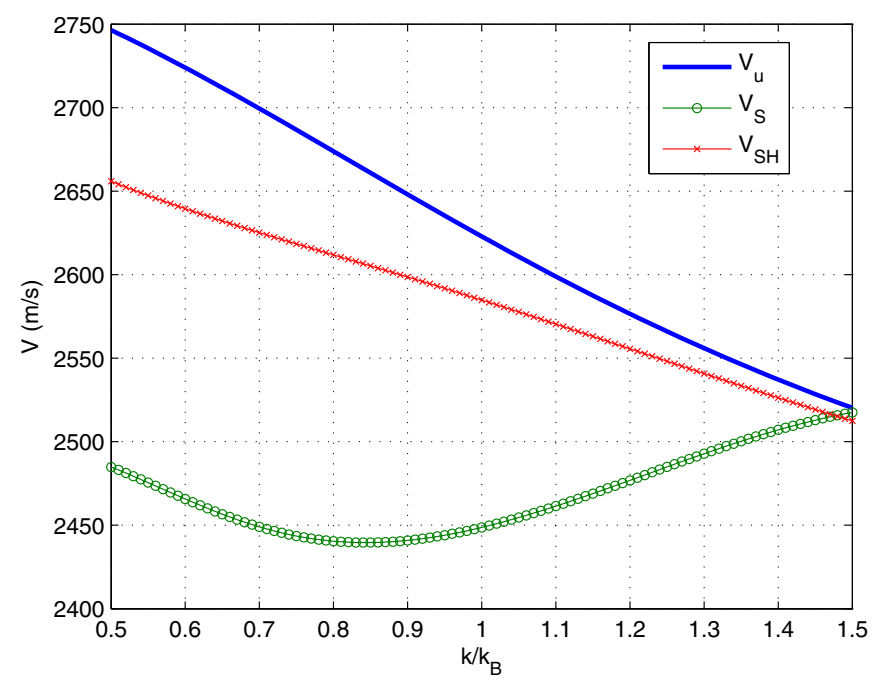

FIG. 7. Phase velocity vs wave number dispersion curves of the S-SAW and SH-SAW propagating along the $X$ axis on the electrically closed surface of half-infinite $\mathrm{BaTiO}_{3} / \mathrm{PZT} 6 \mathrm{~B}$ phononic crystal with a symmetric unit cell. The exterior layer is $\mathrm{BaTiO}_{3}$ of thickness $0.5 h . V_{S}$ is S-SAW velocity, $V_{\mathrm{SH}}$ is SH-SAW velocity, $V_{u}=\omega_{u} / k$ is the limiting wave velocity associated with the upper edge of the lowest stop band. 


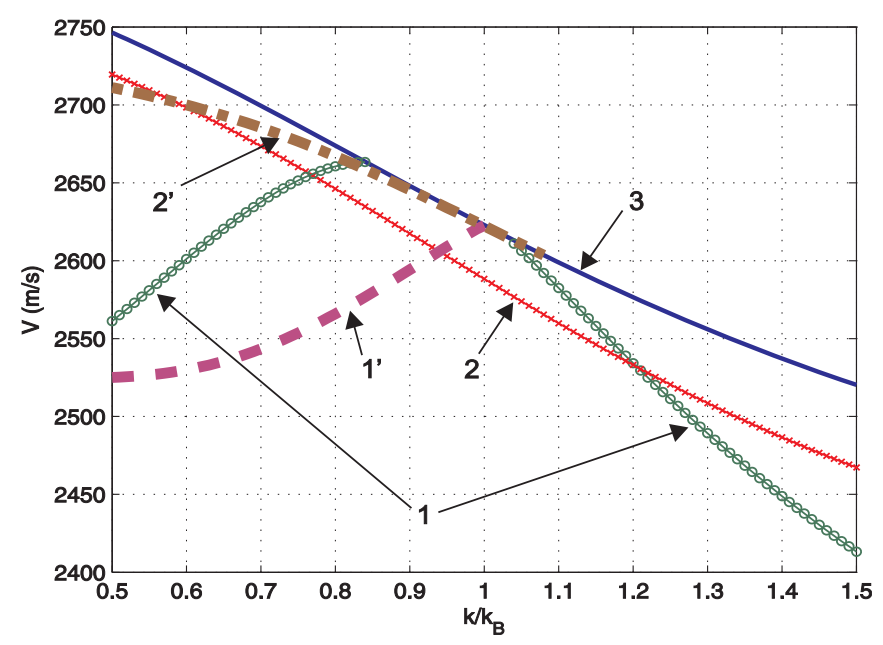

FIG. 8. Phase velocity vs wave number dispersion curves of the S-SAW (curves 1 and $1^{\prime}$ ) and SH-SAW (curves 2 and $2^{\prime}$ ) in the lowest stop band of the phononic crystal with an asymmetric unit cell made of PZT6B and $\mathrm{BaTiO}_{3}$ layers. The surface is electrically closed. Curves 1 and 2 correspond to $\mathrm{PZT} 6 \mathrm{~B} / \mathrm{BaTiO}_{3}$ structure with the exterior PZT6B layer of thickness $0.9 \mathrm{~h}$. Curves $1^{\prime}$ and $2^{\prime}$ correspond to $\mathrm{BaTiO}_{3} / \mathrm{PZT} 6 \mathrm{~B}$ structure with the exterior $\mathrm{BaTiO}_{3}$ layer of thickness $0.8 \mathrm{~h}$. Curve 3 is the limiting wave velocity associated with the upper edge of the lowest stop band.

S-SAW ceases to exist and only the SH-SAW comes about. Further decreasing the thickness of the exterior PZT6B layer enlarges the interval of the S-SAW nonexistence via shifting the right-hand part of the S-SAW dispersion branch (curve 1) to the right edge of the figure. In turn, the SH-SAW dispersion branch (curve 2) locally approaches the curve of limiting velocity (curve 3), touches it, and then, similarly to the S-SAW branch, breaks up into two parts, thus giving rise to the interval of $k$ values where SH-SAW does not exist. Continuing to reduce the PZT6B layer thickness removes PZT6B from the exterior and it is now the $\mathrm{BaTiO}_{3}$ layer which becomes an exterior one. Figure 8 also shows the SAW branches in $\mathrm{BaTiO}_{3} / \mathrm{PZT} 6 \mathrm{~B}$ with the exterior $\mathrm{BaTiO}_{3}$ layer of thickness $0.8 h$ (curves $1^{\prime}$ and $2^{\prime}$ ). In this case, we observe an interval $k / k B>1.1$ where neither S-SAW nor SH-SAW exist in the given lowest stop band. However, the SAWs re-appear one by one when the thickness of the exterior $\mathrm{BaTiO}_{3}$ layer approaches $0.5 h$. Eventually, once it reaches $0.5 h$ and hence the unit cell becomes symmetric, we arrive at the dispersion curves shown in Fig. 7.

\section{CONCLUDING REMARKS}

A series of statements have been proved concerning the admissible number of SAWs per full stop band at fixed wave number $k$ in piezoelectric arbitrarily anisotropic 1D phononic crystals with symmetric unit cell. The latter implies that the layer ordering within a period is invariant with respect to its midplane. The number of SAWs depends on the electrical boundary condition and on whether the stop band is the lowest one or not. The main predictions obtained in the paper are enclosed in Table I. Our derivation circumvents any explicit analytic calculations of the SAW parameters, which are hardly feasible in practice, but develops and employs
TABLE I. Maximum number of SAWs per full stop band at a fixed $k$ in the phononic crystal with symmetric unit cell under different electrical conditions on the mechanically free surface. Surface: e-closed: electrically closed, e-open: electrically open, and e-free: boundary with vacuum. Other columns refer to SAWs in the case of general anisotropy, to piezoactive sagittaly polarized S-SAWs, and to piezoactive SH-SAWs. The notations $\left(0, \omega_{u}\right)$ and $\left(\omega_{l}, \omega_{u}\right)$ imply the lowest and the upper stop bands, respectively.

\begin{tabular}{lcccccc}
\hline \hline & \multicolumn{2}{c}{ SAWs } & \multicolumn{2}{c}{ S-SAWs } & \multicolumn{2}{c}{ SH-SAWs } \\
\hline Surface & $\left(0, \omega_{u}\right)$ & $\left(\omega_{l}, \omega_{u}\right)$ & $\left(0, \omega_{u}\right)$ & $\left(\omega_{l}, \omega_{u}\right)$ & $\left(0, \omega_{u}\right)$ & $\left(\omega_{l}, \omega_{u}\right)$ \\
e-closed & 2 & 2 & 1 & 1 & 1 & 1 \\
e-open & 1 & 2 & 1 & 1 & 0 & 1 \\
e-free & 2 & 2 & 1 & 2 & 1 & 1 \\
\hline \hline
\end{tabular}

instead a certain general approach combining the FloquetBloch formalism and the matrix methods stemming from the theory of SAWs in homogeneous piezoelectric media.

It is instructive to compare the maximum number of SAWs possible per full stop band for a piezoelectric 1D phononic crystal with symmetric unit cell and a similar data for a purely elastic phononic crystal and for a piezoelectric one with an arbitrary (asymmetric) unit cell established in Refs. [46,47], respectively. Generally, the piezoelectric coupling increases this number relatively to the corresponding pure elastic case. The exception is the sagittally polarized SAW: Despite the piezoelectric effect, still only one SAW can exist in any stop band for the electrically closed and open surfaces and in the lowest stop band for the electrically free surface (see Table I), which is the same as in the pure elastic case. On the other hand, the number of SAWs per stop band in a piezoelectric crystal with asymmetric unit cell can exceed their number in a crystal with symmetric unit cell. For instance, the former can support two sagittally polarized piezoactive SAWs in a stop band while the latter cannot. On transforming an asymmetric unit cell to symmetric, e.g., by changing the thickness of the exterior layer, an extra SAW branch may vanish either via coalescing with a stop band edge and then ceasing to exist or via merging in one with another SAW branch.

\section{ACKNOWLEDGMENTS}

The authors thank V. I. Alshits for helpful discussions. The work by A. Darinskii was supported by the Ministry of Science and Higher Education of the Russian Federation.

\section{APPENDIX A}

Let an acoustoelectric wave propagate in a piezoelectric nonconductive medium characterized by the density $\rho$, the elastic stiffness tensor at constant electric field $c_{i j k l}^{E}$, the piezoelectric tensor $e_{i j k}$, and the dielectric permittivity tensor at constant strain $\varepsilon_{i j}^{S}(i, j, k, l=1,2,3)$. In the frame of quasielectrostatic approximation, the mechanical displacement $\mathbf{u}(\mathbf{r}, t)$ and the electrical potential $\varphi(\mathbf{r}, t)$, and hence also the mechanical stress tensor $\hat{\sigma}(\mathbf{r}, t)$ and the electrical displacement $\mathbf{D}(\mathbf{r}, t)$, can be determined by solving the 
equations [61]

$$
\frac{\partial \sigma_{i j}}{\partial x_{j}}=-\rho \omega^{2} u_{i}, \quad \frac{\partial D_{i}}{\partial x_{i}}=0,
$$

with

$$
\sigma_{i j}=c_{i j k l}^{E} \frac{\partial u_{k}}{\partial x_{l}}+e_{k i j} \frac{\partial \varphi}{\partial x_{k}}, \quad D_{i}=e_{i j k} \frac{\partial u_{j}}{\partial x_{k}}-\varepsilon_{i j}^{S} \frac{\partial \varphi}{\partial x_{j}} .
$$

Inserting (A2) into (A1) and assuming the space-time dependence of the form (1) yields a system of four second-order ordinary differential equations on the amplitudes $\mathbf{a}(y)$ and $\phi(y)$,

$$
\text { (nn) } \frac{d^{2}}{d y^{2}}\left(\begin{array}{l}
\mathbf{a} \\
\phi
\end{array}\right)+i k[(m n)+(n m)] \frac{d}{d y}\left(\begin{array}{l}
\mathbf{a} \\
\phi
\end{array}\right)
$$

plugging (1) in Eq. (A2) yields

$$
\left(\begin{array}{l}
\mathbf{l} \\
d
\end{array}\right)=-k(n m)\left(\begin{array}{l}
\mathbf{a} \\
\phi
\end{array}\right)+i(n n) \frac{d}{d y}\left(\begin{array}{l}
\mathbf{a} \\
\phi
\end{array}\right),
$$

where $\mathbf{l}(y)=i \hat{\sigma} \mathbf{n}$ and $d(y)=i \mathbf{D} \cdot \mathbf{n}$. Combining Eqs. (A3) and (A4) and defining the vectors

$$
\boldsymbol{\xi}_{\Phi}(y)=(\mathbf{a}, \phi, \mathbf{l}, d)^{t} \quad \text { or } \boldsymbol{\xi}_{F}(y)=(\mathbf{a}, d, \mathbf{l}, \phi)^{t},
$$

where ${ }^{t}$ means transposition, allows one to replace (A3) with the system of eight first-order differential equations

$$
\frac{d \boldsymbol{\xi}_{P}}{d y}=i \hat{\mathbf{N}}_{P} \boldsymbol{\xi}_{P}, P=\Phi, F
$$

where

$$
\hat{\mathbf{N}}_{\Phi}=-\left(\begin{array}{cc}
k(n n)^{-1}(n m) & (n n)^{-1} \\
k^{2}\left[(m n)(n n)^{-1}(n m)-(m m)\right]+\rho \omega^{2} \hat{\mathbf{I}}^{\prime} & k(m n)(n n)^{-1}
\end{array}\right)
$$

and $\hat{\mathbf{N}}_{F}$ is obtained from the matrix $\hat{\mathbf{N}}_{\Phi}$ by permuting fourth and eighth rows and fourth and eighth columns [48,49]. Note that the Stroh matrix (A7) is written in the form slightly different from that in Refs. [48,49] and is similar to the definition used in Ref. [62] in that it allows us to consider the case $k=0$. The presence of a factor $i$ in the definition of components I and $d$ makes the matrices $\hat{\mathbf{N}}_{P}$ real. Each of them fulfils the symmetry relation

$$
\hat{\mathbf{N}}_{P}=\hat{\mathbf{T}} \hat{\mathbf{N}}_{P}^{t} \hat{\mathbf{T}},
$$

where

$$
\hat{\mathbf{T}}=\left(\begin{array}{cc}
\hat{\mathbf{0}} & \hat{\mathbf{I}} \\
\hat{\mathbf{I}} & \hat{\mathbf{0}}
\end{array}\right),
$$

$\hat{\mathbf{I}}$ and $\hat{\mathbf{0}}$ are the $4 \times 4$ unit and zero matrices, respectively.

\section{APPENDIX B}

In view of symmetry (A8) of $\hat{\mathbf{N}}_{P, i}$ and its real-valuedness, the matrix $\hat{\mathbf{M}}_{P}^{(S)}$ defined by (3) satisfies the equalities

$$
\begin{aligned}
& \hat{\mathbf{M}}_{P}^{(S)}=\hat{\mathbf{T}} \hat{\mathbf{M}}_{P}^{(S) t} \hat{\mathbf{T}}, \\
& \hat{\mathbf{M}}_{P}^{(S)-1}=\hat{\mathbf{M}}_{P}^{(S) *},
\end{aligned}
$$

where $\hat{\mathbf{T}}$ is matrix (A9) and ${ }^{*}$ means complex conjugation. By virtue of Eq. (B2), it follows that $\hat{\mathbf{M}}_{P}^{(S) *} \zeta_{P, \alpha}=\gamma_{\alpha}^{-1} \zeta_{P, \alpha}$. Thus if $\gamma_{\alpha}$ is an eigenvalue of $\hat{\mathbf{M}}_{P}^{(S)}$, then $1 / \gamma_{\alpha}^{*}$ is also an eigenvalue and so the eigenvalues of $\hat{\mathbf{M}}_{P}^{(S)}$ appear pairwise (see Refs. [59,63]), either as

$$
\left|\gamma_{\alpha}\right|=\left|\gamma_{\alpha+4}\right|=1, \quad \alpha=1, \ldots, 4,
$$

or as

$$
\gamma_{\alpha}=\frac{1}{\gamma_{\alpha+4}^{*}}, \quad\left|\gamma_{\alpha}\right| \neq 1, \quad \alpha=1, \ldots, 4 .
$$

Equation (B1) yields the orthonormalization relation of the form

$$
\zeta_{P, \alpha}^{t} \hat{\mathbf{T}} \zeta_{P, \beta}=\delta_{\alpha \beta}, \quad \alpha, \beta=1, \ldots, 8,
$$

where $\delta_{\alpha \beta}$ is the Kronecker symbol. In turn, by (B2), if $\zeta_{P, \alpha}$ is an eigenvector of $\hat{\mathbf{M}}_{P}^{(S)}$ with an eigenvalue $\gamma_{\alpha}$, then $\zeta_{P, \alpha}^{*}$ is its eigenvector with an eigenvalue $1 / \gamma_{\alpha}^{*}$, and so the eigenvectors $\zeta_{P, \alpha}$ and $\zeta_{P, \alpha+4}$, which correspond to a pair of eigenvalues $\gamma_{\alpha}$ and $\gamma_{\alpha+4}$ satisfying (B4), can be introduced in such a way that

$$
\zeta_{P, \alpha}^{*}=\zeta_{P, \alpha+4} .
$$

We are interested in the so-called full stop bands, that is, in frequency intervals where, given the wave number $k$, all eigenvalues occur as pairs fulfilling Eq. (B4). In these intervals, a conjunction of Eqs. (B5) and (B6) is equivalent to the relations

$$
\boldsymbol{\zeta}_{P, \alpha}^{t} \hat{\mathbf{T}} \zeta_{P, \beta}=\delta_{\alpha \beta}, \quad \boldsymbol{\zeta}_{P, \alpha}^{\dagger} \hat{\mathbf{T}} \zeta_{P, \beta}=0, \quad \alpha, \beta=1, \ldots, 4,
$$

where the symbol ${ }^{\dagger}$ stands for the Hermitian conjugation. 
Furthermore, denote by $\hat{\mathbf{U}}_{P}$ and $\hat{\mathbf{V}}_{P}$ the $4 \times 4$ matrices whose columns are the vectors $\mathbf{U}_{P, \alpha}$ and $\mathbf{V}_{P, \alpha}, \alpha=1, \ldots, 4$, introduced by Eq. (5), i.e.,

$$
\begin{aligned}
& \hat{\mathbf{U}}_{P}=\left(\mathbf{U}_{P, 1} \mathbf{U}_{P, 2} \mathbf{U}_{P, 3} \mathbf{U}_{P, 4}\right), \\
& \hat{\mathbf{V}}_{P}=\left(\mathbf{V}_{P, 1} \mathbf{V}_{P, 2} \mathbf{V}_{P, 3} \mathbf{V}_{P, 4}\right) .
\end{aligned}
$$

In terms of matrices (B8), relations (B7) read

$$
\hat{\mathbf{U}}_{P}^{t} \hat{\mathbf{V}}_{P}+\hat{\mathbf{V}}_{P}^{t} \hat{\mathbf{U}}_{P}=\hat{\mathbf{I}}, \quad \hat{\mathbf{U}}_{P}^{\dagger} \hat{\mathbf{V}}_{P}+\hat{\mathbf{V}}_{P}^{\dagger} \hat{\mathbf{U}}_{P}=\hat{\mathbf{0}} .
$$

Referring the matrices (B8) to a full stop band and taking into the chosen numbering of modes, see the discussion around Eq. (6), we introduce the impedance $\hat{\mathbf{Z}}_{P}$ and the admittance $\hat{\mathbf{Y}}_{P}=\hat{\mathbf{Z}}_{P}^{-1}$ of the half-infinite phononic crystal so that

$$
\hat{\mathbf{V}}_{P}=-i \hat{\mathbf{Z}}_{P} \hat{\mathbf{U}}_{P}, \quad \hat{\mathbf{U}}_{P}=i \hat{\mathbf{Y}}_{P} \hat{\mathbf{V}}_{P}
$$

or, in an expanded form,

$$
\mathbf{V}_{P, \alpha}=-i \hat{\mathbf{Z}}_{P} \mathbf{U}_{P, \alpha}, \quad \mathbf{U}_{P, \alpha}=i \hat{\mathbf{Y}}_{P} \mathbf{V}_{P, \alpha}, \quad \alpha=1, \ldots, 4 .
$$

Inserting (B10) in (B9) 2 shows that the impedance and admittance are Hermitian matrices,

$$
\begin{aligned}
& \hat{\mathbf{Z}}_{P}=i \hat{\mathbf{V}}_{P} \hat{\mathbf{U}}_{P}^{-1}=-i \hat{\mathbf{U}}_{P}^{\dagger-1} \hat{\mathbf{V}}_{P}^{\dagger}=\hat{\mathbf{Z}}_{P}^{\dagger}, \\
& \hat{\mathbf{Y}}_{P}=-i \hat{\mathbf{U}}_{P} \hat{\mathbf{V}}_{P}^{-1}=i \hat{\mathbf{V}}_{P}^{\dagger-1} \hat{\mathbf{U}}_{P}^{\dagger}=\hat{\mathbf{Y}}_{P}^{\dagger},
\end{aligned}
$$

which is in agreement with the fact that the energy flux along the $y$ axis is zero.

Important properties of the impedances and admittances follow from the link of their frequency derivative to the kinetic energy and of their value at zero frequency to the internal energy:

$$
\bar{E}_{\text {kin }}==-\frac{\omega}{8} \mathbf{U}_{P}^{\dagger} \frac{\partial \hat{\mathbf{Z}}_{P}}{\partial \omega} \mathbf{U}_{P},\left.\quad \bar{W}\right|_{\omega=0}=\frac{1}{4} \mathbf{U}_{F}^{\dagger} \hat{\mathbf{Z}}_{F} \mathbf{U}_{F},
$$

where $\bar{E}_{\text {kin }}$ is the time-averaged and integrated over depth kinetic energy per unit surface of an arbitrary evanescent wave field generated by the initial data (6), and $\left.\bar{W}\right|_{\omega=0}$ is the integrated over depth internal energy per unit surface of the same wave field in the static limit (see Ref. [47] for more details). Since $\bar{E}_{\text {kin }}>0$ and $\left.\bar{W}\right|_{\omega=0}>0$,

$$
\begin{aligned}
& \partial \hat{\mathbf{Z}}_{P} / \partial \omega \text { are negative definite matrices, } \\
& \partial \hat{\mathbf{Y}}_{P} / \partial \omega \text { are positive definite matrices, }
\end{aligned}
$$

and

$$
\hat{\mathbf{Z}}_{F} \text { and } \hat{\mathbf{Y}}_{F} \text { are positive definite matrices at } \omega=0,
$$

where (B15) takes into account that $\omega=0$ gives rise to a full stop band once $k \neq 0$. Note that $\hat{\mathbf{Z}}_{\Phi}$ is linked via a relation of the type (B13) $)_{2}$ to the electric enthalpy which is not signdefinite, hence neither are the static limits of the matrices $\hat{\mathbf{Z}}_{\Phi}$ and $\hat{\mathbf{Y}}_{\Phi}$.

\section{APPENDIX C}

The matrices $\hat{\mathbf{B}}_{P}$ (9) are linked to the admittances (B12). By manipulating Eq. (B9), we obtain that

$$
\begin{aligned}
\hat{\mathbf{U}}_{P}^{t} \hat{\mathbf{V}}_{P}+\hat{\mathbf{V}}_{P}^{t} \hat{\mathbf{U}}_{P} & =\hat{\mathbf{V}}_{P}^{t}\left(\hat{\mathbf{V}}_{P}^{t-1} \hat{\mathbf{U}}_{P}^{t}+\hat{\mathbf{U}}_{P} \hat{\mathbf{V}}_{P}^{-1}\right) \hat{\mathbf{V}}_{P} \\
& =i \hat{\mathbf{V}}_{P}^{t}\left(\hat{\mathbf{Y}}_{P}^{*}+\hat{\mathbf{Y}}_{P}\right) \hat{\mathbf{V}}_{P}=\mathbf{I},
\end{aligned}
$$

where we have used the Hermiticity $\hat{\mathbf{Y}}_{P}^{t}=\hat{\mathbf{Y}}_{P}^{*}$ of admittances. Equality (C1) and definition (9) yield

$$
\hat{\mathbf{B}}_{P}^{-1}=2 \operatorname{Re}\left(\hat{\mathbf{Y}}_{P}\right) .
$$

Hence

$$
\operatorname{Im}\left(\hat{\mathbf{B}}_{P}\right)=0 \text { and } \hat{\mathbf{B}}_{P}=\hat{\mathbf{B}}_{P}^{t} .
$$

Correspondingly, from Eqs. (B14) and (B15),

$$
\begin{aligned}
& \frac{\partial \hat{\mathbf{B}}_{P}}{\partial \omega} \text { are negative definite matrices } \\
& \text { in the full stop bands, } \\
& \hat{\mathbf{B}}_{F} \text { is a positive definite matrix at } \omega=0 .
\end{aligned}
$$

However,

$$
\begin{aligned}
& \hat{\mathbf{B}}_{\Phi} \text { has three positive and one negative } \\
& \text { eigenvalue at } \omega=0 .
\end{aligned}
$$

The latter property may be proved in two steps. First, writing out the fourth lines in the relations $\mathbf{U}_{P, \alpha}=i \hat{\mathbf{Y}}_{P} \mathbf{V}_{P, \alpha}(\mathrm{B} 10)_{2}$ for $\Phi_{\alpha}$ and $D_{\alpha}$ at $P=\Phi$ and $F$, and then inserting one into the other yields $Y_{\Phi, 44}=-1 / Y_{F, 44}$, where $Y_{P, 44}$ is the 44th element of $\hat{\mathbf{Y}}_{P}$. Hence, by Eq. (C2), $\left(\hat{\mathbf{B}}_{\Phi}^{-1}\right)_{44}=-1 /\left(\hat{\mathbf{B}}_{F}^{-1}\right)_{44}$. In consequence, given the frequency, the matrices $\hat{\mathbf{B}}_{\Phi}$ and $\hat{\mathbf{B}}_{F}$ cannot be either both positive-definite or both negativedefinite and therefore

$$
\begin{aligned}
& \hat{\mathbf{B}}_{\Phi} \text { and } \hat{\mathbf{B}}_{F} \text { cannot have all eight eigenvalues } \\
& \text { of the same sign. }
\end{aligned}
$$

Second, observing that $\hat{\mathbf{B}}_{F}$ and $\hat{\mathbf{B}}_{\Phi}$ have the common upper $3 \times 3$ diagonal block, denoting the eigenvalues of this block by $\tau_{1} \leqslant \tau_{1} \leqslant \tau_{3}$, and ordering the eigenvalues $\tau_{P, i}$ of $\hat{\mathbf{B}}_{P}$ in such a way that $\tau_{P, 1} \leqslant \tau_{P, 2} \leqslant \tau_{P, 3} \leqslant \tau_{P, 4}$, we take advantage of the so-called separation theorem [64], which provides the inequalities

$$
\begin{aligned}
& \tau_{\Phi, 1} \leqslant \tau_{1} \leqslant \tau_{\Phi, 2} \leqslant \tau_{2} \leqslant \tau_{\Phi, 3} \leqslant \tau_{3} \leqslant \tau_{\Phi, 4}, \\
& \tau_{F, 1} \leqslant \tau_{1} \leqslant \tau_{F, 2} \leqslant \tau_{2} \leqslant \tau_{F, 3} \leqslant \tau_{3} \leqslant \tau_{F, 4} .
\end{aligned}
$$

By (C8), if all four $\tau_{\Phi, \alpha}$ are of the same sign, then at least three of $\tau_{F, \alpha}$ are also of this sign; in fact, by (C5), there are exactly three such $\tau_{F, \alpha}$ 's. Similarly, if all $\tau_{F, \alpha}$ are of the same sign, then three $\tau_{\Phi, \alpha}$ are of this sign as well. In particular, by Eq. (C5), all $\tau_{F, \alpha}$ are positive at $\omega=0$, hence statement(C6) holds true.

Note that the matrices $\hat{\mathbf{B}}_{P}$ are finite inside the full stop bands. This fact is not evident at the possible secluded values of $\omega$ and $k$, at which a coincidental degeneracy of eigenvalues of the transfer matrix $\hat{\mathbf{M}}_{P}^{(S)}$ occurs, rendering $\hat{\mathbf{M}}_{P}^{(S)}$ nonsemisimple (not diagonalizable) [59]. In this case, each of the matrices $\hat{\mathbf{B}}_{P}$ seen as sums of dyadic products, which is equivalent to the product of matrices in Eq. (9),

$$
\hat{\mathbf{B}}_{P}=i \sum_{\alpha=1}^{4} \mathbf{V}_{P, \alpha} \otimes \mathbf{V}_{P, \alpha},
$$

contains dyads which diverge on approaching those secluded degeneracy points. However, by analogy with the case of a nonpiezoelectric phononic crystal [46], it can be shown that 
the divergent terms entering individual dyads cancel each other when the dyads are being summed.

In view of $(\mathrm{C} 3)$, the matrices $\hat{\mathbf{B}}_{P}$ have four real eigenvalues $\tau_{P, \alpha}$ and four real eigenvectors $\mathbf{e}_{P, \alpha}$ obeying the relation $\mathbf{e}_{P, \alpha}^{t} \mathbf{e}_{P, \beta}=\delta_{\alpha \beta}, \alpha, \beta=1, \ldots, 4$. Differentiating both sides of the spectral decomposition $\hat{\mathbf{B}}_{P}=\sum_{\alpha=1}^{4} \tau_{P, \alpha} \mathbf{e}_{P, \alpha} \otimes$ $\mathbf{e}_{P, \alpha}$ with respect to $\omega$, then multiplying the result from the left and right by the eigenvector $\mathbf{e}_{P, \alpha}$, and invoking (C4) yields $\mathbf{e}_{P, \alpha}^{t}\left(\partial \hat{\mathbf{B}}_{P} / \partial \omega\right) \mathbf{e}_{P, \alpha}=\partial \tau_{P, \alpha} / \partial \omega<0$. Bearing in mind that the matrices $\hat{\mathbf{B}}_{P}$ are finite within the full stop band, we conclude that

\section{the eigenvalues of $\hat{\mathbf{B}}_{P}$ monotonically decrease}

with increasing frequency in the full stop bands.

A SAW frequency $\omega_{P}$, at which the boundary conditions (7) or (8) are fulfilled, is a simple zero of $\operatorname{det} \hat{\mathbf{V}}_{P}$, i.e. locally $\operatorname{det} \hat{\mathbf{V}}_{P} \propto \omega-\omega_{P}$. This is because, by (B14), $\omega_{P}$ is also a zero of $\operatorname{det} \hat{\mathbf{Z}}_{P}$, which must be its simple zero by virtue of Eq. (B14). Hence, $\omega_{P}$ is a double zero of $\operatorname{det} \hat{\mathbf{B}}_{P} \propto\left(\omega-\omega_{P}\right)^{2}$, which in view of Eq. (C7) can be the case only due to a pair of eigenvalues of $\hat{\mathbf{B}}_{P}$ vanishing simultaneously. Thus

$$
\begin{aligned}
& \text { the eigenvalues of each of the matrices } \mathbf{B}_{F} \text { and } \mathbf{B}_{\Phi} \\
& \text { in the full stop bands vanish pairwise. }
\end{aligned}
$$

According to Sec. III A, at most two SAWs can occur on a mechanically free electrically closed or open surface. Assume that two SAWs on the electrically closed surface do come about at some frequencies $\omega_{F 1} \leqslant \omega_{F 2}$ lying in a given full stop band $\omega_{l}<\omega<\omega_{u}$. This means that all four eigenvalues $\tau_{F, \alpha}$ of $\hat{\mathbf{B}}_{F}$ are positive at $\omega<\omega_{F 1}$ and all four of them are negative at $\omega>\omega_{F 2}$. Then, by virtue of the property formulated below Eq. (C8), three eigenvalues $\tau_{\Phi, \alpha}$ of $\hat{\mathbf{B}}_{\Phi}(\alpha=2,3,4)$ are positive at $\omega_{l}<\omega<\omega_{F 1}$ and three eigenvalues $\tau_{\Phi, \alpha}(\alpha=1,2,3)$ are negative at $\omega_{F 2}<\omega<\omega_{u}$. Thus

$$
\text { a pair } \tau_{\Phi, 2} \text { and } \tau_{\Phi, 3} \text { changes simultaneously sign }
$$$$
\text { at some } \omega_{\Phi} \text { such that } \omega_{F 1} \leqslant \omega_{\Phi} \leqslant \omega_{F 2} \text {. }
$$

Analogously it can be shown that if two SAWs on the electrically open surface come about at some frequencies $\omega_{\Phi 1} \leqslant$ $\omega_{\Phi 2}$ in a given full stop band, then

$$
\begin{aligned}
& \text { a pair of the eigenvalues of } \hat{\mathbf{B}}_{F} \text { changes } \\
& \text { simultaneously sign at some } \omega_{F} \\
& \text { such that } \omega_{\Phi 1} \leqslant \omega_{F} \leqslant \omega_{\Phi 2} .
\end{aligned}
$$

The analysis of the SAW existence on the mechanically clamped surface is based on the properties of the matrices $\hat{\mathbf{Q}}$ defined by Eq. (13) (see Sec. III D). By analogy with the proof of Eq. (C1), it can be shown that $\hat{\mathbf{Q}}_{P}^{-1}=-2 \operatorname{Re}\left(\hat{\mathbf{Z}}_{P}\right)$. Hence in view of (B14) 2 and (B15),

$$
\begin{aligned}
& \partial \hat{\mathbf{Q}}_{P} / \partial \omega \text { are negative definite matrices } \\
& \text { in the full stop bands; } \\
& \hat{\mathbf{Q}}_{F} \text { has four negative eigenvalues at } \omega=0 ; \\
& \hat{\mathbf{Q}}_{\Phi} \text { has three negative and one positive } \\
& \text { eigenvalue at } \omega=0 .
\end{aligned}
$$

Like $\hat{\mathbf{B}}_{P}$, the matrices $\hat{\mathbf{Q}}_{P}$ remain finite inside the full stop bands. In addition the eigenvalues of $\hat{\mathbf{Q}}_{P}$ also vanish only pairwise. These properties lead us to the statements formulated in Sec. III D.

At the stop band edges $\omega_{u}$ and $\omega_{l} \neq 0$, two eigenvalues $\gamma_{\alpha}$ and $\gamma_{\alpha+4}=1 / \gamma_{\alpha}^{*}$ of the transfer matrix $\hat{\mathbf{M}}_{P}^{(S)}$ degenerate into one eigenvalue $\gamma_{d}$ with $\left|\gamma_{d}\right|=1$ and two corresponding eigenvectors $\zeta_{P, \alpha}$ and $\zeta_{P, \alpha+4}$ coalesce into one eigenvector $\zeta_{P, d}=\left(\mathbf{U}_{P, d}, \mathbf{V}_{P, d}\right)^{t}$, so that $\hat{\mathbf{M}}_{P}^{(S)}$ becomes nonsemisimple. By analogy with the terminology of the SAW theory for homogeneous half-spaces $[48,49]$, the mode generated by the eigenvector $\zeta_{P, d}$ may be called limiting. As opposed to a similar degeneracy strictly inside a stop band [see a discussion around Eq. (C9)], the matrix $\hat{\mathbf{B}}$ represented as dyadic sum (C9) generally contains only one dyad diverging at $\omega \rightarrow \omega_{l}+0$ or $\omega \rightarrow \omega_{u}-0$ and therefore the divergent terms do not cancel out, leading to the divergence of $\hat{\mathbf{B}}_{P}$. More specifically, the perturbation theory similar to that of the nonpiezoelectric case [46] shows that at least one eigenvalue of $\hat{\mathbf{B}}_{P}$ tends to minus infinity or plus infinity as $\omega \rightarrow \omega_{u}-0$ or $\omega \rightarrow \omega_{l}+0$, respectively, unless the exceptional possibility that $\mathbf{V}_{P, d}=\mathbf{0}$, i.e., that the limiting wave alone satisfies the corresponding boundary condition. Thus we arrive at the SAW existence criterion formulated at the end of Sec. III A.
[1] Acoustic Metamaterials and Phononic Crystals, edited by P. A. Deymier (Springer, Heidelberg, 2013).

[2] V. Laude, Phononic Crystals: Artificial Crystals for Sonic, Acoustic, and Elastic Waves, Studies in Mathematical Physics Vol. 26 (De Gruyter, 2015).

[3] Phononic Crystals: Fundamentals and Applications, edited by A. Khelif and A. Adibi (Springer, New York, 2016).

[4] E. H. El Boudouti and B. Djafari-Rouhani, One-Dimensional Phononic Crystals, in Acoustic Metamaterials and Phononic Crystals, edited by P. A. Deymier (Springer, Heidelberg, 2013), p. 45.
[5] V. I. Alshits and A. L. Shuvalov, Bragg reflection of sound in a periodic structure of piezoelectric-crystal layers with superconducting or metallized interlayers, Zh. Eksp. Teor. Fiz. 103, 1356 (1993) [JETP 76, 663 (1993)].

[6] V. I. Alshits and A. L. Shuvalov, Resonance reflection and transmission of shear elastic waves in multilayered piezoelectric structures, J. Appl. Phys. 77, 2659 (1995).

[7] A. L. Shuvalov and A. S. Gorkunova, Transverse acoustic waves in piezoelectric and ferroelectric antiphase superlattices, Phys. Rev. B 59, 9070 (1999). 
[8] E. H. El Boudouti, B. Djafari-Rouhani, A. Akjouj, and L. Dobrzynski, Acoustic waves in solid and fluid layered materials, Surf. Sci. Rep. 64, 471 (2009).

[9] Y. El Hassouani, E. H. El Boudouti, B. Djafari-Rouhani, H. Aynaou, and L. Dobrzynski, Surface and interface acoustic waves in solid-fluid superlattices: Green's function approach, Phys. Rev. B 74, 144306 (2006).

[10] I. Quotane, E. H. El Boudouti, and B. Djafari-Rouhani, Trapped-mode-induced Fano resonance and acoustical transparency in a one- dimensional solid-fluid phononic crystal, Phys. Rev. B 97, 024304 (2018).

[11] F. Nori and J. P. Rodriguez, Acoustic and electronic properties of one-dimensional quasicrystals, Phys. Rev. B 34, 2207 (1986).

[12] S. Tamura and J. P. Wolfe, Acoustic-phonon transmission in quasiperiodic superlattices, Phys. Rev. B 36, 3491(R) (1987).

[13] Y.-Y. Zhu, N.-B. Ming, and W.-H. Jiang, Ultrasonic spectrum in Fibonacci acoustic superlattices, Phys. Rev. B 40, 8536 (1989).

[14] I. Quotane, E. H. El Boudouti, B. Djafari-Rouhani, Y. El Hassouani, and V. R. Velasco, Bulk and surface acoustic waves in solid- fluid Fibonacci layered materials, Ultrasonics 61, 40 (2015).

[15] R. E. Camley, B. Djafari-Rouhani, L. Dobrzynski, and A. A. Maradudin, Transverse elastic waves in periodically layered infinite and semi-infinite media, Phys. Rev. B 27, 7318 (1983).

[16] E. H. El Boudouti, B. Djafari-Rouhani, E. M. Khourdifi, and L. Dobrzynski, Surface and interface elastic waves in superlattices: transverse localized and resonant modes, Phys. Rev. B 48, 10987 (1993).

[17] E. H. El Boudouti, B. Djafari-Rouhani, A. Akjouj, and L. Dobrzynski, Theory of surface and interface transverse elastic waves in $N$-layer superlattices, Phys. Rev. B 54, 14728 (1996).

[18] A. Bousfia, E. H. El Boudouti, D. Bria, and A. Nougaoui, Surface and interface shear horizontal acoustic waves in piezoelectric superlattices, J. Appl. Phys. 87, 4507 (2000).

[19] S.-Y. Ren and Y.-C. Chang, Theory of confinement effects in finite one-dimensional phononic crystals, Phys. Rev. B 75, 212301 (2007).

[20] S. Chen, S. Lin, and Z. Wong, Shear horizontal surface acoustic waves in semi-infinite piezoelectrics/metal superlattices, Ultrasonics 49, 446 (2009).

[21] Ph. Gatignol, C. Potel, and J. F. de Belleval, Two families of modal waves for periodic structures with two field functions: a Cayleigh-Hamilton approach, Acustica-Acta Acustica 93, 959 (2007).

[22] A. L. Shuvalov, O. Poncelet, and S. V. Golkin, Existence and spectral properties of shear horizontal surface waves in vertically periodic half-spaces, Proc. R. Soc. Lond. Ser. A 465, 1489 (2009).

[23] M. Alami, E. H. El Boudouti, B. Djafari-Rouhani, Y. El Hassouani, and A. Talbi, Surface acoustic waves in onedimensional piezoelectric-metallic phononic crystal: Effect of a cap layer, Ultrasonics 90, 80 (2018).

[24] B. Djafari-Rouhani, L. Dobrzynski, O. Hardouin Duparc, R. E. Camley, and A. A. Maradudin, Sagittal elastic waves in infinite and semi-infinite superlattices, Phys. Rev. B 28, 1711 (1983).

[25] A. Nougaoui and B. Djafari-Rouhani, Elastic waves in periodically layered infinite and semi-infinite anisotropic media, Surf. Sci. 185, 125 (1987).
[26] A. N. Podlipenets and N. A. Shul'ga, Numerical study of the propagation of Rayleigh and Lamb waves in orthotropic periodic structures, Int. Appl. Mech. 23, 1013 (1987).

[27] E. H. El Boudouti, B. Djafari-Rouhani, and A. Nougaoui, Surface elastic waves in superlattices: Sagittal localized and resonant modes, Phys. Rev. B 51, 13801 (1995).

[28] A. Nougaoui and B. Djafari-Rouhani, Vibrations in elastic and piezoelectric superlattices, J. Electron. Spectrosc. Relat. Phenom. 45, 197 (1987).

[29] S. Chen, Y. Zhang, S. Lin, and Z. Fu, Study on the electromechanical coupling coefficient of Rayleigh-type surface acoustic waves in semi-infinite piezoelectrics/non-piezoelectrics superlattices, Ultrasonics 54, 604 (2014).

[30] A. Nougaoui and B. Djafari-Rouhani, Complex band structure of acoustic waves in superlattices, Surf. Sci. 199, 623 (1988).

[31] B. Honein, A. M. B. Braga, P. Barbone, and G. Herrmann, Wave propagation in piezoelectric layered media with some applications, J. Intell. Mater. Syst. Struct. 2, 542 (1991).

[32] T. Aono and S. Tamura, Surface and pseudosurface acoustic waves in superlattices, Phys. Rev. B 58, 4838 (1998).

[33] Y. Tanaka and S.-i. Tamura, Surface acoustic waves in twodimensional periodic elastic structures, Phys. Rev. B 58, 7958 (1998); Acoustic stop bands of surface and bulk modes in two-dimensional phononic lattices consisting of aluminium and a polymer, 60, 13294 (1999).

[34] B. Manzanares-Martinez and F. Ramos-Mendieta, Surface elastic waves in solid composites of two-dimensional periodicity, Phys. Rev. B 68, 134303 (2003).

[35] T.-T. Wu, Z.-G. Huang, and S. Lin, Surface and bulk acoustic waves in two-dimensional phononic crystals consisting of materials with general anisotropy, Phys. Rev. B 69, 094301 (2004).

[36] V. Laude, M. Wilm, S. Benchabane, and A. Khelif, Full band gap for surface acoustic waves in a piezoelectric phononic crystal, Phys. Rev. E 71, 036607 (2005).

[37] T. T. Wu, Z. C. Hsu, and Z. G. Huang, Band gaps and electromechanical coupling coefficient of a surface acoustic wave in a two-dimensional piezoelectric phononic crystal, Phys. Rev. B 71, 064303 (2005).

[38] S. Benchabane, A. Khelif, J.-Y. Rauch, L. Robert, and V. Laude, Evidence for complete surface wave band gap in a piezoelectric phononic crystal, Phys. Rev. E 73, 065601(R) (2006).

[39] J. H. Sun and T. T. Wu, "Propagation of surface acoustic waves through sharply bent two-dimensional phononic crystal waveguides using a finite difference time-domain method, Phys. Rev. B 74, 174305 (2006).

[40] Y. Tanaka, Y. Takafumi, and S. Tamura, Surface guided waves in two-dimensional phononic crystals, Wave Motion 44, 501 (2007).

[41] D. Zhao, Z. Liu, C. Qiu, Z. He, F. Cai, and M. Ke, Surface acoustic waves in two-dimensional phononic crystals: Dispersion relation and the eigenfield distribution of surface modes, Phys. Rev. B 76, 144301 (2007).

[42] Z.-Z. Yan and Y.-S. Wang, Calculation of band structures for surface waves in two-dimensional phononic crystals with a wavelet-based method, Phys. Rev. B 78, 094306 (2008).

[43] Y. Li, Z. Hou, M. Oudich, and M. B. Assouar, Analysis of surface acoustic wave propagation in a two-dimensional phononic crystal, J. Appl. Phys. 112, 023524 (2012).

[44] A. A. Kutsenko and A. L. Shuvalov, Shear surface waves in phononic crystals, J. Acoust. Soc. Am. 133, 653 (2013). 
[45] D. Yudistira, A. Boes, B. Djafari-Rouhani, Y. Pennec, L.-Y. Yeo, A. Mitchell, and J. R. Friend, Monolithic Phononic Crystals with a Surface Acoustic Band Gap from Surface PhononPolariton Coupling, Phys. Rev. Lett. 113, 215503 (2014).

[46] A. N. Darinskii and A. L. Shuvalov, Surface acoustic waves on one-dimensional phononic crystals of general anisotropy: Existence considerations, Phys. Rev. B 98, 024309 (2018).

[47] A. N. Darinskii and A. L. Shuvalov, Existence of surface acoustic waves on half-infinite one-dimensional piezoelectric phononic crystals of general anisotropy, Phys. Rev. B. 99, 174305 (2019).

[48] J. Lothe and D. M. Barnett, Integral formalism for surface waves in piezoelectric crystals. Existence considerations, J. Appl. Phys. 47, 1799 (1976).

[49] J. Lothe and D. M. Barnett, Further development of the theory for surface waves in piezoelectric crystals, Phys. Norvegica 8 , 239 (1977).

[50] Yu. I. Sirotin and M. P. Shaskol'skaya, Fundamentals of Crystal Physics (Mir, Moscow, 1982).

[51] A. N. Darinskii and A. L. Shuvalov, Non-leaky surface acoustic waves in the passbands of one-dimensional phononic crystals, Ultrasonics 98, 108 (2019).

[52] Y.-Y. Zhu, X.-J. Zhang, Y.-Q. Lu, Y.-F. Chen, S.-N. Zhu, and N.B. Ming, New Type of Polariton in a Piezoelectric Superlattice, Phys. Rev. Lett. 90, 053903 (2003).

[53] Xue-jin Zhang, Ran-qi Zhu, Jun Zhao, Yan-feng Chen, and Y.-Y. Zhu, Phonon-polariton dispersion and the polariton-based photonic band gap in piezoelectric superlattices, Phys. Rev. B 69, 085118 (2004).
[54] Z. Liu and W. Zhang, Scaling and localization of polaritonic states in piezoelectric Fibonacci superlattices, Phys. Rev. B 72, 134304 (2005).

[55] Y. Chao, J. Sheng, J. A. Sedlacek, and J. P. Shaffer, Surface phonon polaritons on anisotropic piezoelectric superlattices, Phys. Rev. B 93, 045419 (2016).

[56] S. V. Biryukov, Yu. V. Gulyaev, V. V. Krylov, and V. P. Plessky, Surface Acoustic Waves in Inhomogeneous Media (SpringerVerlag, Berlin, 1995).

[57] A. N. Darinskii, E. Le Clezio, and G. Feuillard, Acoustic waves in the vicinity of the normal to the surface of piezoelectric crystals, IEEE Trans. Ultrason., Ferroelect., Freq. Contr. 54, 612 (2007).

[58] A. N. Darinskii, E. Le Clezio, and G. Feuillard, The role of electromagnetic waves in the reflection of acoustic waves in piezoelectric crystals, Wave Motion 45, 428 (2008).

[59] M. C. Pease III, Methods of Matrix Algebra (Academic Press, New York, 1965).

[60] J. Yang, An Introduction to the Theory of Piezoelectricity (Springer Science + Business Media inc., Boston, 2005).

[61] B. A. Auld, Acoustic Fields and Waves in Solids (Krieger, Malabar, FL, 1990), Vols. I and II.

[62] K. A. Ingebrigtsen and A. Tonning, Elastic surface waves in crystals, Phys. Rev. 184, 942 (1969).

[63] A. M. B. Braga and G. Herrmann, Floquet waves in anisotropic periodically layered composites, J. Acoust. Soc. Am. 91, 1211 (1992).

[64] J. H. Wilkinson, The Algebraic Eigenvalue Problem (Oxford University Press, New York, 1965). 